\title{
A MULTI-LEVEL FAST MULTiPole BEM FOR 3-D ELASTODYNAMICS IN THE FREQUENCY DOMAIN
}

\author{
Stéphanie CHAILLAT ${ }^{1,2}$, Marc BONNET ${ }^{1 *}$, Jean-François SEMBLAT $^{2}$ \\ ${ }^{1}$ Solid Mechanics Laboratory (UMR CNRS 7649), Department of Mechanics \\ École Polytechnique, F-91128 Palaiseau cedex, France \\ chaillat,bonnet@1ms.polytechnique.fr \\ ${ }^{2}$ Université Paris-Est, Laboratoire Central des Ponts et Chaussées \\ 58 boulevard Lefebvre, 75015 Paris, France \\ semblat@lcpc.fr
}

\begin{abstract}
To reduce computational complexity and memory requirement for 3-D elastodynamics using the boundary element method (BEM), a multi-level fast multipole BEM (FM-BEM) is proposed. The diagonal form for the expansion of the elastodynamic fundamental solution is used, with a truncation parameter adjusted to the subdivision level, a feature necessary for achieving optimal computational efficiency. Both the single-level and multi-level forms of the elastodynamic FM-BEM are considered, with emphasis on the latter. Crucial implementation issues, including the truncation of the multipole expansion, the optimal number of levels, the direct and inverse extrapolation steps are examined in detail with the backing of numerical experiments. A complexity analysis for both the single-level and multi-level versions is conducted. The correctness and computational performances of the proposed elastodynamic FMM are demonstrated on numerical examples, featuring up to $O\left(10^{6}\right)$ DOFs run on a single-processor PC and including the diffraction of an incident $P$ plane wave by a semi-spherical or semi-ellipsoidal canyon, representative of topographic site effects.
\end{abstract}

Keywords: Fast multipole method; Boundary element method; 3-D elastodynamics; Topographic site effects

\section{Introduction}

The boundary element method (BEM), pioneered in the sixties [6,41], is a mesh reduction method, subject to restrictive constitutive assumptions but yielding highly accurate solutions. It is in particular well suited to deal with unbounded-domain idealizations commonly used in e.g. acoustics [50], electromagnetics $[35,39]$ or seismology [7, 22]. In contrast with domain discretization methods, artificial boundary conditions [18] are not needed for dealing with the radiation conditions, and grid dispersion cumulative effects are absent $[24,51]$.

However, in traditional boundary element (BE) implementations, the dimensional advantage with respect to domain discretization methods is offset by the fully-populated nature of the BEM coefficient matrix, with set-up and solution times rapidly increasing with the problem size $N$. It is thus essential to develop alternative, faster strategies that allow to still exploit the known advantages of BEMs when large $N$ prohibit the use of traditional implementations. Fast BEMs, i.e. BEMs of complexity lower than that of traditional BEMs, appeared around 1985 with an iterative integral-equation

Computer Methods in Applied Mechanics and Engineering, 197:4233-4249 (2008)

* Corresponding author 
approach for solving 2-D Laplace problems within $O(N)$ CPU time per iteration [42]. The fast multipole method (FMM) concept was introduced in [19,20], in the context of many-particle simulations. The FMM then naturally led to fast multipole boundary element methods (FM-BEMs), whose scope and capabilities have rapidly progressed, especially in connection with application in electromagnetics [21, 31, 32, 53], but also in other fields including acoustics $[14,36,48]$ and computational mechanics [30]. Many of these investigations are summarized in a review article by Nishimura [37]. The FMM, as well as other fast BEM approaches [23, 27, 55, 56], intrinsically relies upon an iterative solution approach for the linear system of discretized BEM equations, with solution times typically of order $O(N \log N)$ per iteration for frequency-domain wave propagation problems (instead of $O\left(N^{2}\right)$ per iteration with traditional forms of the BEM).

With a view toward future applications in seismology and dynamic soil-structure interaction, this article is concerned with the formulation and implementation of a multi-level FM-BEM for 3-D elastodynamics in the frequency domain. Only a few references address this particular area of application. Two- and three-dimensional FM-BEMs for frequency-domain elastodynamics are proposed in [16] and $[17,58]$, respectively, while time-domain problems are addressed in [54]. As the free-space fundamental solution used in elastodynamic boundary integral formulations is expressed in terms of the full-space Green's function for the scalar Helmholtz equation and its derivatives, many of the existing developments towards fast integral solvers for equations of the Helmholtz type (including in particular the Maxwell equations) are transposable to elastodynamic BEMs. A complete presentation of an elastodynamic FM-BEM formulation based on such transposition is the main purpose of this article. In particular, computational efficiency of fast elastodynamic BEMs in the mid-frequency regime is enhanced by using the so-called diagonal form for the Helmholtz Green's function [11,43-45]; the upper limit stems from the fact that the size $N$ becomes intractable at high frequencies, while the diagonal form breaks down at very low frequencies and must be replaced with other types of expansions [5, 10, 25]. Improving on [17], where the diagonal form was already adopted, the present work implements crucial features such as the adjustment of the truncation parameter in the multipole expansion to the subdivision level, known from recent studies for the Maxwell equations such as $[8,25]$ to be necessary for achieving optimal computational efficiency. Both the single-level and multi-level forms of the FM-BEM are considered, with emphasis on the latter. A substantial fraction of the article is then devoted to the discussion, backed with the results of numerical experiments, of crucial implementation details (many of which transposing methods previously proposed for electromagnetic FM-BEMs $[8,53]$ to the present 3-D elastodynamic context) and a complexity analysis for both the single-level and multi-level versions.

The article is organized as follows. Classical concepts pertaining to elastodynamic BEMs are recalled in Section 2. Then, Section 3 presents underlying motivations and fundamental concepts for the elastodynamic FMM. Next, several crucial computational and implementation issues are addressed in Section 4. Section 5 is devoted to the analysis and numerical verification of the algorithmic complexity of single-level and multi-level versions. Finally, the correctness and computational performances of the proposed FM-BEM are assessed in Section 6 on numerical examples involving up to $N=O\left(10^{6}\right)$ nodal unknowns. The latter include the diffraction of an incident $\mathrm{P}$ plane wave by a semi-spherical or semi-ellipsoidal canyon, representative of topographic site effects.

\section{Boundary element method}

\subsection{Boundary integral representation}

Let $\Omega \subset \mathbb{R}^{3}$ denote the region of space occupied by a three-dimensional elastic solid with isotropic constitutive properties defined by $\mu$ (shear modulus), $\nu$ (Poisson's ratio) and $\rho$ (mass density). Timeharmonic motions, with circular frequency $\omega$, induced by a prescribed traction distribution $\boldsymbol{t}^{D}$ on 
the boundary $\partial \Omega$ and in the absence of body forces are considered for definiteness in this article. The accommodation of other boundary conditions needs only minor modifications to the treatment proposed therein. The displacement $\boldsymbol{u}$ is given at an interior point $\boldsymbol{x} \in \Omega$ by the following well-known representation formula [3]:

$$
u_{k}(\boldsymbol{x})=-\int_{\partial \Omega} u_{i}(\boldsymbol{y}) T_{i}^{k}(\boldsymbol{x}, \boldsymbol{y} ; \omega) d S_{y}+\int_{\partial \Omega} t_{i}^{D}(\boldsymbol{y}) U_{i}^{k}(\boldsymbol{x}, \boldsymbol{y} ; \omega) d S_{y} \quad(\boldsymbol{x} \in \Omega),
$$

where $U_{i}^{k}(\boldsymbol{x}, \boldsymbol{y} ; \omega)$ and $T_{i}^{k}(\boldsymbol{x}, \boldsymbol{y} ; \omega)$ denote the $i$-th components of the elastodynamic fundamental solution, i.e. of the displacement and traction, respectively, generated at $\boldsymbol{y} \in \mathbb{R}^{3}$ by a unit point force applied at $\boldsymbol{x} \in \mathbb{R}^{3}$ along the direction $k$, given by [12]:

$$
\begin{aligned}
U_{i}^{k}(\boldsymbol{x}, \boldsymbol{y} ; \omega) & =\frac{1}{k_{\mathrm{S}}^{2} \mu}\left(\left(\delta_{q s} \delta_{i k}-\delta_{q k} \delta_{i s}\right) \frac{\partial}{\partial x_{q}} \frac{\partial}{\partial y_{s}} G\left(|\boldsymbol{y}-\boldsymbol{x}| ; k_{\mathrm{S}}\right)+\frac{\partial}{\partial x_{i}} \frac{\partial}{\partial y_{k}} G\left(|\boldsymbol{y}-\boldsymbol{x}| ; k_{\mathrm{P}}\right)\right), \\
T_{i}^{k}(\boldsymbol{x}, \boldsymbol{y} ; \omega) & =C_{i j h \ell} \frac{\partial}{\partial y_{\ell}} U_{h}^{k}(\boldsymbol{x}, \boldsymbol{y} ; \omega) n_{j}(\boldsymbol{y}),
\end{aligned}
$$

in which $k_{\mathrm{S}}$ and $k_{\mathrm{P}}$ are the respective wavenumbers of $\mathrm{S}$ and $\mathrm{P}$ elastic waves, so that

$$
k_{\mathrm{S}}^{2}=\frac{\rho \omega^{2}}{\mu}, \quad k_{\mathrm{P}}=\gamma k_{\mathrm{S}}, \quad \gamma^{2}=\frac{1-2 \nu}{2(1-\nu)},
$$

$G(\cdot ; k)$ is the free-space Green's function for the Helmholtz equation with wavenumber $k$, given by

$$
G(r ; k)=\frac{\exp (\mathrm{i} k r)}{4 \pi r}
$$

$\boldsymbol{n}(\boldsymbol{y})$ is the unit normal to $\partial \Omega$ directed outwards of $\Omega$, and $C_{i j h \ell}$ are the components of the fourthorder elasticity tensor, i.e.:

$$
C_{i j h \ell}=\mu\left[\frac{2 \nu}{1-2 \nu} \delta_{i j} \delta_{h \ell}+\delta_{i h} \delta_{j \ell}+\delta_{j h} \delta_{i \ell}\right]
$$

\subsection{Boundary integral equation}

When $\boldsymbol{x} \in \partial \Omega$, a singularity occurs in $\boldsymbol{y}=\boldsymbol{x}$. With the help of a well-documented limiting process [4], the integral representation (1) yields the integral equation:

$$
(\mathcal{K} u)(\boldsymbol{x})=f(\boldsymbol{x}) \quad(\boldsymbol{x} \in \partial \Omega),
$$

with the linear integral operator $\mathcal{K}$ and the right-hand side $f$ defined by

$$
\begin{aligned}
(\mathcal{K} u)(\boldsymbol{x}) & =c_{i k}(\boldsymbol{x}) u_{i}(\boldsymbol{x})+(\text { P.V. }) \int_{\partial \Omega} u_{i}(\boldsymbol{y}) T_{i}^{k}(\boldsymbol{x}, \boldsymbol{y} ; \omega) d S_{y} \\
f(\boldsymbol{x}) & =\int_{\partial \Omega} t_{i}^{D}(\boldsymbol{y}) U_{i}^{k}(\boldsymbol{x}, \boldsymbol{y} ; \omega) d S_{y}
\end{aligned}
$$

where (P.V.) indicates a Cauchy principal value (CPV) singular integral and the free-term $c_{i k}(\boldsymbol{x})$ is equal to $0.5 \delta_{i k}$ in the usual case where $\partial \Omega$ is smooth at $\boldsymbol{x}$. The integral operator (7) may be recast into alternative, equivalent regularized forms which are free of CPV integrals [3]. Equations (1) and (6) are applicable to either interior or exterior elastodynamic problems. 


\subsection{Boundary Element Method}

The numerical solution of boundary integral equation (6) is based on a discretization of the surface $\partial \Omega$ into $N_{E}$ isoparametric boundary elements. Piecewise-linear interpolation of displacements, based on three-noded triangular boundary elements, is used in this work. The $N_{I}$ displacement interpolation nodes thus defined also serve as collocation points. This discretization process transforms (6) into a square complex-valued matrix equation of size $N=3 N_{I}$ of the form

$$
[K]\{u\}=\{f\}
$$

where the $N$-vector $\{u\}$ collects the sought degrees of freedom (DOFs), namely the nodal displacement components, while the $N \times N$ matrix of influence coefficients $[K]$ and the $N$-vector $\{f\}$ arise from (7) and (8), respectively. Setting up the matrix $[K]$ classically requires the computation of all element integrals for each collocation point, thus needing a computational time of order $O\left(N^{2}\right)$.

\subsection{Solution strategy for the BEM equations}

The influence matrix $[K]$ is fully-populated. Storing $[K]$ is thus limited, on ordinary computers, to BEM models of size not exceeding $N=O\left(10^{4}\right)$. Direct solvers, such as the LU factorization, require $O\left(N^{3}\right)$ arithmetic operations (i.e. they have a $O\left(N^{3}\right)$ complexity), and are thus also limited to moderately-sized BEM models. BEM problems of larger size are preferably solved by means of iterative algorithms (GMRES [47] being the usual choice), which build sequences of solution candidates until convergence within a predefined tolerance is reached. With reference to (9), each GMRES iteration requires one evaluation of $[K]\{u\}$ for given $\{u\}$, a task requiring a computing time of order $O\left(N^{2}\right)$ if either $[K]$ is stored or $[K]\{u\}$ is evaluated by means of standard BEM numerical integration procedures. In the latter case, the $O\left(N^{2}\right)$ complexity stems from the fact that, again, all element integrals must be recomputed for each collocation point. Applications of the BEM to large models (typically $N=O\left(10^{6}\right)$ ) require evaluation procedures for $[K]\{u\}$ that are fast (i.e. of complexity below $O\left(N^{2}\right)$ ) and that avoid explicit formation and storage of $[K]$. The fast multipole method (FMM) is known in many other fields as a very efficient approach for achieving these objectives. It is therefore chosen as the basis for the present formulation and implementation of a fast elastodynamic BEM.

\section{Fast Multipole Method: principle}

\subsection{Multipole expansions of the elastodynamic fundamental solutions}

The FMM is based on a reformulation of the fundamental solutions in terms of products of functions of $\boldsymbol{x}$ and of $\boldsymbol{y}$. This allows to re-use integrations with respect to $\boldsymbol{y}$ when the collocation point $\boldsymbol{x}$ is changed, thereby lowering the $O\left(N^{2}\right)$ complexity per iteration entailed by standard BEMs. The elastodynamic fundamental solutions $(2 \mathrm{a}, \mathrm{b})$ are linear combinations of derivatives of the Green's function (4) for the Helmholtz equation. On recasting the position vector $\boldsymbol{r}=\boldsymbol{y}-\boldsymbol{x}$ in the form

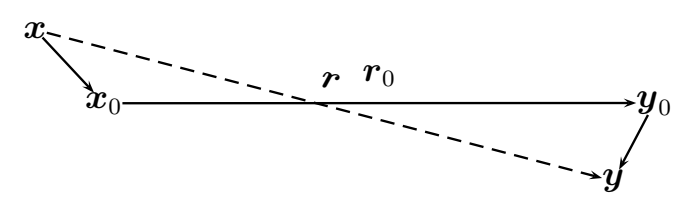

Figure 1: Decomposition of the position vector: notation. 
$\boldsymbol{r}=\boldsymbol{r}_{0}+\left(\boldsymbol{y}-\boldsymbol{y}_{0}\right)-\left(\boldsymbol{x}-\boldsymbol{x}_{0}\right)$, where $\boldsymbol{x}_{0}$ and $\boldsymbol{y}_{0}$ are two poles and $\boldsymbol{r}_{0}=\boldsymbol{y}_{0}-\boldsymbol{x}_{0}$ (Fig. 1), the Helmholtz Green's function is shown $[9,11]$ to admit the decomposition

$$
G(|\boldsymbol{r}| ; k)=\lim _{L \rightarrow+\infty} \int_{\hat{\boldsymbol{s}} \in S} e^{\mathrm{i} k \hat{\boldsymbol{s}} \cdot\left(\boldsymbol{y}-\boldsymbol{y}_{0}\right)} \mathcal{G}_{L}\left(\hat{\boldsymbol{s}} ; \boldsymbol{r}_{0} ; k\right) e^{-\mathrm{i} k \hat{\boldsymbol{s}} .\left(\boldsymbol{x}-\boldsymbol{x}_{0}\right)} \mathrm{d} \hat{\boldsymbol{s}}
$$

where $S$ is the unit sphere of $\mathbb{R}^{3}$ and the transfer function $\mathcal{G}_{L}\left(\hat{\boldsymbol{s}} ; \boldsymbol{r}_{0} ; k\right)$ is defined in terms of the Legendre polynomials $P_{p}$ and the spherical Hankel functions of the first kind $h_{p}^{(1)}$ by:

$$
\mathcal{G}_{L}\left(\hat{\boldsymbol{s}} ; \boldsymbol{r}_{0} ; k\right)=\frac{\mathrm{i} k}{16 \pi^{2}} \sum_{0 \leq p \leq L}(2 p+1) \mathrm{i}^{p} h_{p}^{(1)}\left(k\left|\boldsymbol{r}_{0}\right|\right) P_{p}\left(\cos \left(\hat{\boldsymbol{s}}, \boldsymbol{r}_{0}\right)\right) .
$$

The decomposition (10)-(11) is seen to achieve the desired separation of variables $\boldsymbol{x}$ and $\boldsymbol{y}$. Then, to recast the elastodynamic fundamental solutions in a form similar to (10)-(11), one simply notes that (10) implies:

$$
\frac{\partial}{\partial x_{i}} G(|\boldsymbol{r}| ; k)=-\mathrm{i} k \hat{s}_{i} G(|\boldsymbol{r}| ; k), \quad \frac{\partial}{\partial y_{i}} G(|\boldsymbol{r}| ; k)=\mathrm{i} k \hat{s}_{i} G(|\boldsymbol{r}| ; k),
$$

where $\hat{s}_{i}$ is the $i$-th component of the vector $\hat{s}$. Then, on substituting (12) into (2a,b) and invoking decomposition (10)-(11), the following multipole decomposition of the elastodynamic fundamental solutions is obtained:

$$
\begin{aligned}
U_{i}^{k}(\boldsymbol{x}, \boldsymbol{y} ; \omega)= & \lim _{L \rightarrow+\infty} \int_{\hat{\boldsymbol{s}} \in S} e^{\mathrm{i} k_{\mathrm{P}} \hat{\boldsymbol{s}} \cdot\left(\boldsymbol{y}-\boldsymbol{y}_{0}\right)} \mathcal{U}_{i, L}^{k, \mathrm{P}}\left(\hat{\boldsymbol{s}} ; \boldsymbol{r}_{0}\right) e^{-\mathrm{i} k_{\mathrm{P}} \hat{\boldsymbol{s}} \cdot\left(\boldsymbol{x}-\boldsymbol{x}_{0}\right)} \mathrm{d} \hat{\boldsymbol{s}} \\
& +\lim _{L \rightarrow+\infty} \int_{\hat{\boldsymbol{s}} \in S} e^{\mathrm{i} k_{\mathrm{S}} \hat{\boldsymbol{s}} \cdot\left(\boldsymbol{y}-\boldsymbol{y}_{0}\right)} \mathcal{U}_{i, L}^{k, \mathrm{~S}}\left(\hat{\boldsymbol{s}} ; \boldsymbol{r}_{0}\right) e^{-\mathrm{i} k_{\mathrm{S}} \hat{\boldsymbol{s}} \cdot\left(\boldsymbol{x}-\boldsymbol{x}_{0}\right)} \mathrm{d} \hat{\boldsymbol{s}}, \\
T_{i}^{k}(\boldsymbol{x}, \boldsymbol{y} ; \omega)= & \lim _{L \rightarrow+\infty} \int_{\hat{\boldsymbol{s}} \in S} e^{\mathrm{i} k_{\mathrm{P}} \hat{\boldsymbol{s}} \cdot\left(\boldsymbol{y}-\boldsymbol{y}_{0}\right)} \mathcal{T}_{i, L}^{k, \mathrm{P}}\left(\hat{\boldsymbol{s}} ; \boldsymbol{r}_{0}\right) e^{-\mathrm{i} k_{\mathrm{P}} \hat{\boldsymbol{s}} \cdot\left(\boldsymbol{x}-\boldsymbol{x}_{0}\right)} \mathrm{d} \hat{\boldsymbol{s}} \\
& +\lim _{L \rightarrow+\infty} \int_{\hat{\boldsymbol{s}} \in S} e^{\mathrm{i} k_{\mathrm{s}} \hat{\boldsymbol{s}} \cdot\left(\boldsymbol{y}-\boldsymbol{y}_{0}\right)} \mathcal{T}_{i, L}^{k, \mathrm{~S}}\left(\hat{\boldsymbol{s}} ; \boldsymbol{r}_{0}\right) e^{-\mathrm{i} k_{\mathrm{S}} \hat{\boldsymbol{s}} \cdot\left(\boldsymbol{x}-\boldsymbol{x}_{0}\right)} \mathrm{d} \hat{\boldsymbol{s}},
\end{aligned}
$$

with the elastodynamic transfer functions given in terms of the acoustic transfer function $\mathcal{G}_{L}$ by

$$
\begin{gathered}
\mathcal{U}_{i, L}^{k, \mathrm{P}}\left(\hat{\boldsymbol{s}} ; \boldsymbol{r}_{0}\right)=\frac{\gamma^{2}}{\mu} \hat{s}_{i} \hat{s}_{k} \mathcal{G}_{L}\left(\hat{\boldsymbol{s}} ; \boldsymbol{r}_{0} ; k_{\mathrm{P}}\right), \\
\mathcal{T}_{i, L}^{k, \mathrm{P}}\left(\hat{\boldsymbol{s}} ; \boldsymbol{r}_{0}\right)=\frac{\mathrm{i} k_{\mathrm{S}} \gamma^{3}}{\mu} C_{i j h \ell} \hat{s}_{\ell} \hat{s}_{h} \hat{s}_{k} \mathcal{G}_{L}\left(\hat{\boldsymbol{s}} ; \boldsymbol{r}_{0} ; k_{\mathrm{P}}\right) n_{j}(\boldsymbol{y}), \\
\mathcal{U}_{i, L}^{k, \mathrm{~S}}\left(\hat{\boldsymbol{s}} ; \boldsymbol{r}_{0}\right)=\frac{1}{\mu}\left(\delta_{i k}-\hat{s}_{k} \hat{s}_{i}\right) \mathcal{G}_{L}\left(\hat{\boldsymbol{s}} ; \boldsymbol{r}_{0} ; k_{\mathrm{S}}\right), \\
\mathcal{T}_{i, L}^{k, \mathrm{~S}}\left(\hat{\boldsymbol{s}} ; \boldsymbol{r}_{0}\right)=\frac{\mathrm{i} k_{\mathrm{S}}}{\mu}\left(\delta_{h k}-\hat{s}_{k} \hat{s}_{h}\right) C_{i j h \ell} \hat{s}_{\ell} \mathcal{G}_{L}\left(\hat{\boldsymbol{s}} ; \boldsymbol{r}_{0} ; k_{\mathrm{S}}\right) n_{j}(\boldsymbol{y}) .
\end{gathered}
$$

Truncation error and clustering. In practice, the limiting process $L \rightarrow+\infty$ in (10) or (13), (14) cannot be performed exactly and is replaced with an evaluation for a suitably chosen finite value of $L$. A key error analysis result [9] states that there exist four constants $C_{1}, C_{2}, C_{3}, C_{4}$ such that

$$
\begin{aligned}
L=C_{1}+C_{2} k\left|\boldsymbol{r}-\boldsymbol{r}_{0}\right| & +C_{3} \ln \left(k\left|\boldsymbol{r}-\boldsymbol{r}_{0}\right|\right)+C_{4} \ln \epsilon^{-1} \\
& \Longrightarrow\left|\frac{\exp (\mathrm{i} k|\boldsymbol{r}|)}{4 \pi|\boldsymbol{r}|}-\int_{\hat{\boldsymbol{s}} \in S} e^{\mathrm{i} k \hat{\boldsymbol{s}} \cdot\left(\boldsymbol{y}-\boldsymbol{y}_{0}\right)} \mathcal{G}_{L}\left(\hat{\boldsymbol{s}} ; \boldsymbol{r}_{0} ; k\right) e^{-\mathrm{i} k \hat{\boldsymbol{s}} \cdot\left(\boldsymbol{x}-\boldsymbol{x}_{0}\right)} \mathrm{d} \hat{\boldsymbol{s}}\right|<\epsilon
\end{aligned}
$$




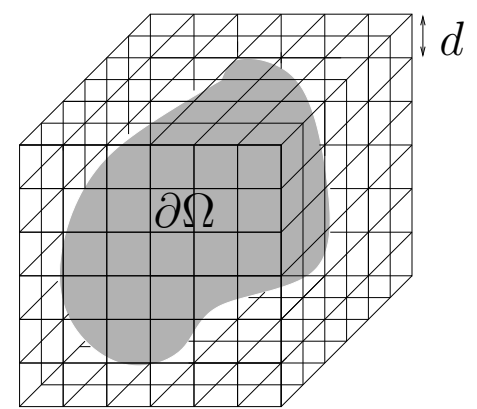

Figure 2: $3 D$ cubic grid embedding the boundary $\partial \Omega$.

for any chosen error level $\epsilon<1$, whenever

$$
\left|\boldsymbol{r}-\boldsymbol{r}_{0}\right| /\left|\boldsymbol{r}_{0}\right|=\left|\left(\boldsymbol{y}-\boldsymbol{y}_{0}\right)-\left(\boldsymbol{x}-\boldsymbol{x}_{0}\right)\right| /\left|\boldsymbol{r}_{0}\right| \leq 2 / \sqrt{5}
$$

The error bound (17), (18) implies that expansions (13), (14) must be used for well-separated sets of collocation and integration points clustered around poles $\boldsymbol{x}_{0}$ and $\boldsymbol{y}_{0}$. Moreover, (17) also indicates that the value of the truncation parameter $L$ allowing to achieve a given level of accuracy $\epsilon$ increases with the size of these clusters. Other studies on error control in multipole expansions for Helmholtz equations can be found in e.g. [21,38].

\subsection{Single-level fast multipole formulation}

In the single-level FMM, a 3D cubic grid of linear spacing $d$ embedding the boundary $\partial \Omega$ is introduced (Fig. 2). The centers of the cubic cells thus defined are taken as poles $\boldsymbol{x}_{0}$ or $\boldsymbol{y}_{0}$ in decompositions (13), (14). Two cells are deemed adjacent if they have at least one common point, e.g. a vertex (Fig. 3). Whenever $\boldsymbol{x}$ and $\boldsymbol{y}$ belong to cells $\mathcal{C}_{x}, \mathcal{C}_{y}$ that are not adjacent, condition (18) is automatically fulfilled (as one then always has $\left|\boldsymbol{r}-\boldsymbol{r}_{0}\right| /\left|\boldsymbol{r}_{0}\right| \leq \sqrt{3} / 2<2 / \sqrt{5}$ ) and expansions (13), (14) can be safely used. Conversely, when $\boldsymbol{x}$ and $\boldsymbol{y}$ lie in adjacent cells, condition (18) is not assured and the classical expressions $(2 \mathrm{a}, \mathrm{b})$ of the fundamental solutions are used instead. These considerations lead to reformulate expressions (7) and (8), for any collocation point $\boldsymbol{x}$ lying in a given cell $\mathcal{C}_{x}$, as

$$
\begin{aligned}
(\mathcal{K} u)(\boldsymbol{x}) & =(\mathcal{K} u)^{\text {near }}(\boldsymbol{x})+(\mathcal{K} u)^{\mathrm{FM}}(\boldsymbol{x}), \\
f(\boldsymbol{x}) & =f^{\text {near }}(\boldsymbol{x})+f^{\mathrm{FM}}(\boldsymbol{x})
\end{aligned}
$$

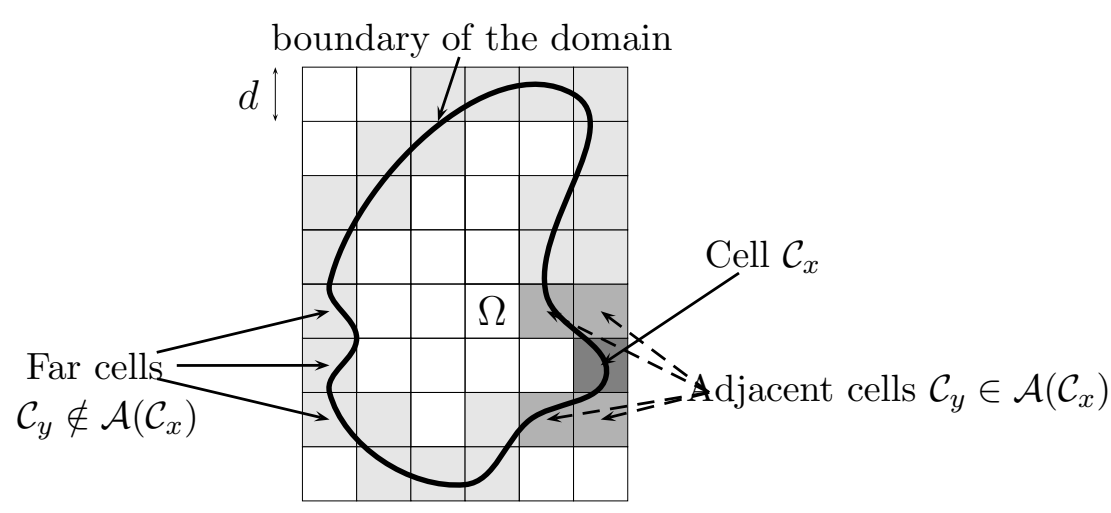

Figure 3: Definition of the adjacent cells 
where, letting $\mathcal{A}(\mathcal{C})$ denote the set of cells which are adjacent to a given cubic cell $\mathcal{C}$ (Fig. 3), the "near" parts are defined for each collocation point $\boldsymbol{x}$ as the net contributions from the portion of boundary situated in cells adjacent to that containing $\boldsymbol{x}$, i.e. by

$$
\begin{aligned}
(\mathcal{K} u)^{\text {near }}(\boldsymbol{x}) & =c_{i k}(\boldsymbol{x}) u_{i}(\boldsymbol{x})+\sum_{\mathcal{C}_{y} \in \mathcal{A}\left(\mathcal{C}_{x}\right)}(\text { P.V. }) \int_{\partial \Omega \cap \mathcal{C}_{y}} u_{i}(\boldsymbol{y}) T_{i}^{k}(\boldsymbol{x}, \boldsymbol{y} ; \omega) d S_{y}, \\
f^{\text {near }}(\boldsymbol{x}) & =\sum_{\mathcal{C}_{y} \in \mathcal{A}\left(\mathcal{C}_{x}\right)} \int_{\partial \Omega \cap \mathcal{C}_{y}} t_{i}^{D}(\boldsymbol{y}) U_{i}^{k}(\boldsymbol{x}, \boldsymbol{y} ; \omega) d S_{y} .
\end{aligned}
$$

The "FM" parts then collect all contributions from cells that are not adjacent to $\mathcal{C}_{x}$ :

$$
\begin{aligned}
(\mathcal{K} u)^{\mathrm{FM}}(\boldsymbol{x}) & =\sum_{\mathcal{C}_{y} \notin \mathcal{A}\left(\mathcal{C}_{x}\right)} \int_{\partial \Omega \cap \mathcal{C}_{y}} u_{i}(\boldsymbol{y}) T_{i}^{k}(\boldsymbol{x}, \boldsymbol{y} ; \omega) d S_{y}, \\
f^{\mathrm{FM}}(\boldsymbol{x}) & =\sum_{\mathcal{C}_{y} \notin \mathcal{A}\left(\mathcal{C}_{x}\right)} \int_{\partial \Omega \cap \mathcal{C}_{y}} t_{i}^{D}(\boldsymbol{y}) U_{i}^{k}(\boldsymbol{x}, \boldsymbol{y} ; \omega) d S_{y} .
\end{aligned}
$$

The "near" contributions $(20 \mathrm{a}, \mathrm{b})$ are evaluated by means of standard BE techniques. The treatment of the "FM" contributions (21a,b) exploits expansions (13), (14) truncated at a finite $L$ and in a manner suggested by their multiplicative form, i.e. (i) evaluate integrals over each cell $\mathcal{C}_{y}$ and associate obtained values to the cell center $\boldsymbol{y}_{0}$, (ii) apply transfer functions to obtain quantities associated to the center $\boldsymbol{x}_{0}$ of cell $\mathcal{C}_{x}$, and (iii) evaluate contribution at each collocation point $\boldsymbol{x} \in \mathcal{C}_{x}$. Accordingly, multipole moments, defined by

$$
\begin{aligned}
\mathcal{R}_{k}^{\mathrm{S}, u}\left(\hat{\boldsymbol{s}} ; \mathcal{C}_{y}\right)= & -\mathrm{i} k_{\mathrm{S}}\left[\delta_{i k} \hat{s}_{j}+\delta_{j k} \hat{s}_{i}-2 \hat{s}_{i} \hat{s}_{j} \hat{s}_{k}\right] \int_{\partial \Omega \cap \mathcal{C}_{y}} u_{i}(\boldsymbol{y}) n_{j}(\boldsymbol{y}) e^{\mathrm{i} k_{\mathrm{S}} \hat{\boldsymbol{s}} \cdot\left(\boldsymbol{y}-\boldsymbol{y}_{0}\right)} d S_{\tilde{y}} \\
\mathcal{R}^{\mathrm{P}, u}\left(\hat{\boldsymbol{s}} ; \mathcal{C}_{y}\right)= & -\mathrm{i} k_{\mathrm{S}} \gamma^{3}\left[\frac{2 \nu}{1-2 \nu} \delta_{i j}+2 \hat{s}_{i} \hat{s}_{j}\right] \int_{\partial \Omega \cap \mathcal{C}_{y}} u_{i}(\boldsymbol{y}) n_{j}(\boldsymbol{y}) e^{\mathrm{i} k_{\mathrm{P}} \hat{\boldsymbol{s}} \cdot\left(\boldsymbol{y}-\boldsymbol{y}_{0}\right)} d S_{\tilde{y}} \\
& \mathcal{R}_{k}^{\mathrm{S}, t}\left(\hat{\boldsymbol{s}} ; \mathcal{C}_{y}\right)=\frac{1}{\mu}\left[\delta_{k a}-\hat{s}_{k} \hat{s}_{a}\right] \int_{\partial \Omega \cap \mathcal{C}_{y}} t_{a}(\boldsymbol{y}) e^{\mathrm{i} k_{\mathrm{S}} \hat{\boldsymbol{s}} \cdot\left(\boldsymbol{y}-\boldsymbol{y}_{0}\right)} d S_{\tilde{y}} \\
& \mathcal{R}^{\mathrm{P}, t}\left(\hat{\boldsymbol{s}} ; \mathcal{C}_{y}\right)=\frac{\gamma^{2}}{\mu} \int_{\partial \Omega \cap \mathcal{C}_{y}} \hat{s}_{a} t_{a}(\boldsymbol{y}) e^{\mathrm{i} k_{\mathrm{P}} \hat{\boldsymbol{s}} \cdot\left(\boldsymbol{y}-\boldsymbol{y}_{0}\right)} d S_{\tilde{y}}
\end{aligned}
$$

are computed for each cell $\mathcal{C}_{y}$ (step (i)). Then, local expansions for the cell $\mathcal{C}_{x}$ are evaluated by applying the transfer functions to the multipole moments according to

$$
\begin{aligned}
& \mathcal{L}_{k}^{\mathrm{S}, u}\left(\hat{\boldsymbol{s}} ; \mathcal{C}_{x}\right)=\sum_{\mathcal{C}_{y} \notin \mathcal{A}\left(\mathcal{C}_{x}\right)} \mathcal{G}_{L}\left(\hat{\boldsymbol{s}} ; \boldsymbol{r}_{0} ; k_{\mathrm{S}}\right) \mathcal{R}_{k}^{\mathrm{S}, u}\left(\hat{\boldsymbol{s}} ; \mathcal{C}_{y}\right), \\
& \mathcal{L}^{\mathrm{P}, u}\left(\hat{\boldsymbol{s}} ; \mathcal{C}_{x}\right)=\sum_{\mathcal{C}_{y} \notin \mathcal{A}\left(\mathcal{C}_{x}\right)} \mathcal{G}_{L}\left(\hat{\boldsymbol{s}} ; \boldsymbol{r}_{0} ; k_{\mathrm{P}}\right) \mathcal{R}^{\mathrm{P}, u}\left(\hat{\boldsymbol{s}} ; \mathcal{C}_{y}\right) \\
& \mathcal{L}_{k}^{\mathrm{S}, t}\left(\hat{\boldsymbol{s}} ; \mathcal{C}_{x}\right)=\sum_{\mathcal{C}_{y} \notin \mathcal{A}\left(\mathcal{C}_{x}\right)} \mathcal{G}_{L}\left(\hat{\boldsymbol{s}} ; \boldsymbol{r}_{0} ; k_{\mathrm{S}}\right) \mathcal{R}_{k}^{\mathrm{S}, t}\left(\hat{\boldsymbol{s}} ; \mathcal{C}_{y}\right), \\
& \mathcal{L}^{\mathrm{P}, t}\left(\hat{\boldsymbol{s}} ; \mathcal{C}_{x}\right)=\sum_{\mathcal{C}_{y} \notin \mathcal{A}\left(\mathcal{C}_{x}\right)} \mathcal{G}_{L}\left(\hat{\boldsymbol{s}} ; \boldsymbol{r}_{0} ; k_{\mathrm{P}}\right) \mathcal{R}^{\mathrm{P}, t}\left(\hat{\boldsymbol{s}} ; \mathcal{C}_{y}\right),
\end{aligned}
$$

where $\boldsymbol{r}_{0}=\boldsymbol{y}_{0}-\boldsymbol{x}_{0}$ joins the centers of cells $\mathcal{C}_{x}$ and $\mathcal{C}_{y}$ (step (ii)). Upon multiplying (24a,b), (25a,b) by the local factors $\exp \left[\mathrm{i} k_{\alpha} \hat{\boldsymbol{s}}\right.$. $\left.\left(\boldsymbol{x}-\boldsymbol{x}_{0}\right)\right]$ (step (iii)) and replacing the integration over the unit sphere in (13), (14) by a numerical quadrature rule based on a set of $Q$ quadrature points $\hat{\boldsymbol{s}}_{q} \in S$ and weights $w_{q}$ (see Section 4.3), the "FM" contributions finally take the form

$$
(\mathcal{K} u)_{k}^{\mathrm{FM}}(\boldsymbol{x}) \approx \sum_{q=1}^{Q} w_{q}\left[e^{-\mathrm{i} k_{\mathrm{S}} \hat{\boldsymbol{s}}_{q} \cdot\left(\boldsymbol{x}-\boldsymbol{x}_{0}\right)} \mathcal{L}_{k}^{\mathrm{S}, u}\left(\hat{\boldsymbol{s}}_{q} ; \mathcal{C}_{x}\right)+e^{-\mathrm{i} k_{\mathrm{P}} \hat{\boldsymbol{s}}_{q} \cdot\left(\boldsymbol{x}-\boldsymbol{x}_{0}\right)}\left(\hat{\boldsymbol{s}}_{q}\right)_{k} \mathcal{L}^{\mathrm{P}, u}\left(\hat{\boldsymbol{s}}_{q} ; \mathcal{C}_{x}\right)\right]
$$



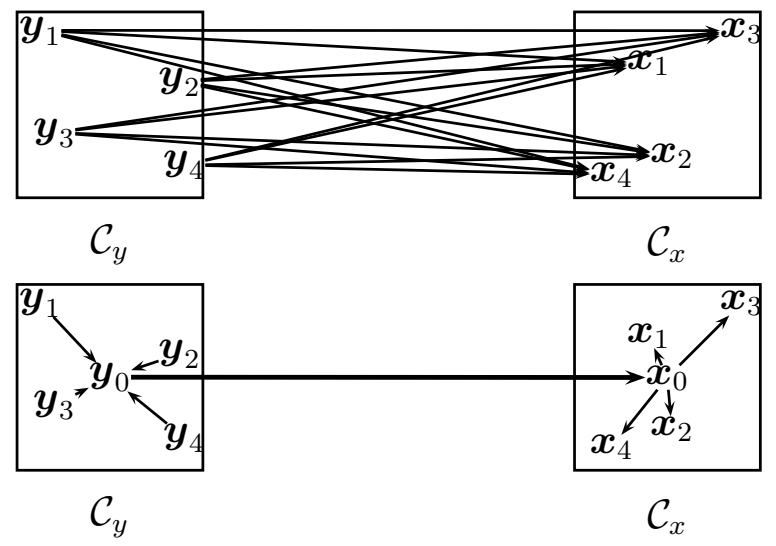

Figure 4: Matrix-vector product without FMM (top) and with FMM (bottom).

$$
f_{k}^{\mathrm{FM}}(\boldsymbol{x}) \approx \sum_{q=1}^{Q} w_{q}\left[e^{-\mathrm{i} k_{\mathrm{S}} \hat{\boldsymbol{s}}_{q} \cdot\left(\boldsymbol{x}-\boldsymbol{x}_{0}\right)} \mathcal{L}_{k}^{\mathrm{S}, t}\left(\hat{\boldsymbol{s}}_{q} ; \mathcal{C}_{x}\right)+e^{-\mathrm{i} k_{\mathrm{P}} \hat{\boldsymbol{s}}_{q} \cdot\left(\boldsymbol{x}-\boldsymbol{x}_{0}\right)}\left(\hat{\boldsymbol{s}}_{q}\right)_{k} \mathcal{L}^{\mathrm{P}, t}\left(\hat{\boldsymbol{s}}_{q} ; \mathcal{C}_{x}\right)\right]
$$

Expression (26) defines the "FM" contribution to the matrix-vector product $[K]\{u\}$, and hence is evaluated once per GMRES iteration, while (27) provides the "FM" contribution to the right-hand side $\{f\}$ and is computed once, prior to calling the GMRES solver. Figure 4 schematically depicts the acceleration mechanism achieved by the previously described steps.

As remarked in section 3.1, the truncation parameter $L$, and hence the maximum degree of Legendre polynomials featured in the transfer functions $\mathcal{G}_{L}\left(\hat{\boldsymbol{s}} ; \boldsymbol{r}_{0} ; k_{\alpha}\right)$, increases with the cell size $d$. Consequently, the number $Q$ of quadrature points necessary for achieving a given accuracy in (26), (27) is also an increasing function of $L$, i.e. of $d$ (see section 4.1 for further elaboration).

The single-level elastodynamic FMM is more efficient than the classical BEM, with a complexity of $O\left(N^{3 / 2}\right)$ per GMRES iteration (as shown in section 5.1). Further acceleration is achievable by adopting a multi-level approach, as described next for the present context of 3-D elastodynamics.

\subsection{Multi-level fast multipole formulation}

To have maximal efficiency, FM-BEM algorithms must confine non-FM calculations to the smallest possible portion of the boundary while clustering whenever possible the computation of influence terms into the largest possible non-adjacent groups. This is achieved by the multi-level FMM [8, 30, $37,52,53$, which is based on using large cells and hierarchically subdividing each cell into $2 \times 2 \times$ $2=8$ children cubic cells. This cell-subdivision approach is systematized by means of an oct-tree structure of cells. The level $\ell=0$, composed of only one cubic cell containing the whole surface $\partial \Omega$, is the tree root. The level- 0 cell is divided into $2 \times 2 \times 2=8$ children cubic cells, which constitute the level $\ell=1$. All level-1 cells being adjacent, the FMM cannot be applied to them. The level $\ell=2$ is then defined by dividing each level- 1 cell into 8 children cells, and so contains 64 cells. The subdivision process is further repeated until the finest level $\ell=\bar{\ell}$, implicitly defined by a preset subdivision-stopping criterion, is reached. Level $-\bar{\ell}$ cells are usually termed leaf cells. The FMM is applied from level $\ell=2$ to level $\ell=\bar{\ell}$, i.e. features $\bar{\ell}-1$ "active" levels.

The multi-level approach basically consists in first applying the FMM to all influence computations between disjoint level-2 cells (so as to use the largest clusters whenever possible), and then recursively tracing the tree downwards, applying the FMM to all interaction between disjoint level- $\ell$ cells that are children of adjacent level- $(\ell-1)$ cells (Fig. 5). Finally, interactions between adjacent leaf cells are treated using traditional (i.e. non FM-based) BE techniques. This approach thus minimizes 


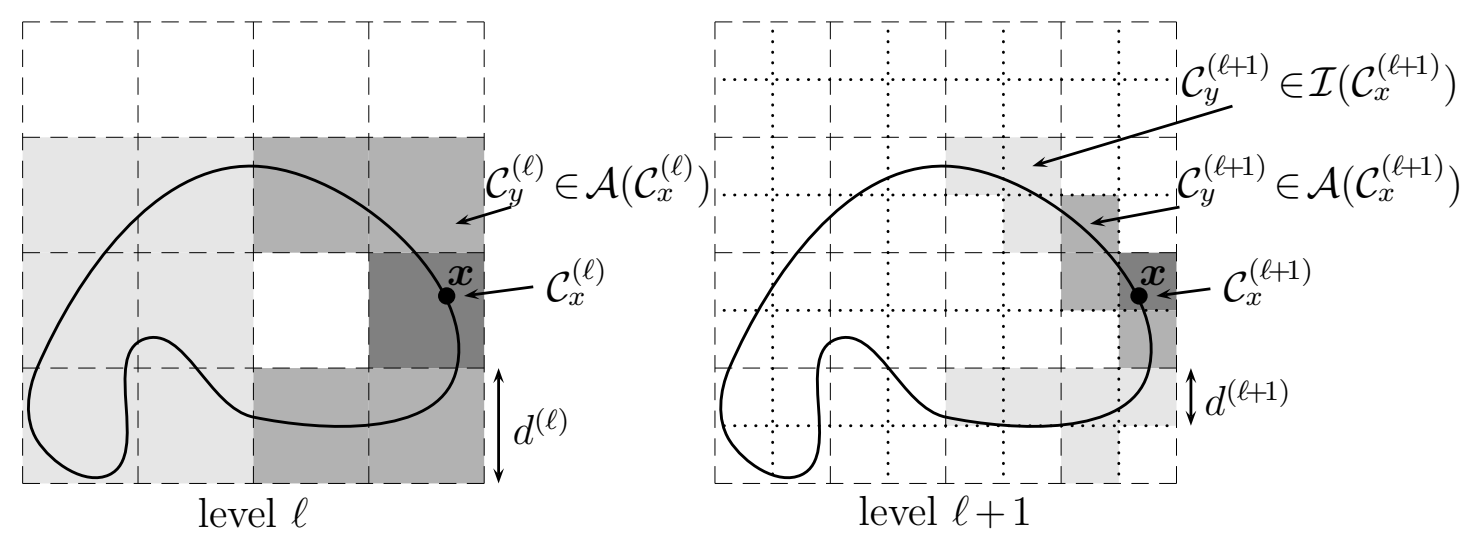

Figure 5: Multi-level fast multipole algorithm. Only multipole moments from non-adjacent (lightgrey) cells $\mathcal{C}_{y}^{(\ell)} \notin \mathcal{A}\left(\mathcal{C}_{x}^{(\ell)}\right)$ may provide (through transfer) FM-computed contributions to $(\mathcal{K} u)^{F M}(\boldsymbol{x})$ at collocation points $\boldsymbol{x}$ lying in cell $\mathcal{C}_{x}^{(\ell)}$. Upon cell subdivision (right), new FM-computed contributions to collocation points in cell $\mathcal{C}_{x}^{(\ell+1)}$ originate from cells $\mathcal{C}_{y}^{(\ell+1)}$ in the interaction list $\mathcal{I}\left(\mathcal{C}_{x}^{(\ell+1)}\right)$ of $\mathcal{C}_{x}^{(\ell+1)}$, while the adjacent region $\mathcal{A}\left(\mathcal{C}_{x}^{(\ell+1)}\right)$ reduces in size.

the overall proportion of influence computations requiring the traditional treatment.

The computation of the discretized linear operator (7), i.e. of the matrix-vector product $[K]\{u\}$, by the multi-level elastodynamic FMM hence consists of the following main steps:

1. Initialization: compute multipole moments $(22 \mathrm{a}, \mathrm{b})$ for all lowest-level cells $\mathcal{C}_{y}=\mathcal{C}_{y}^{\bar{\ell}}$.

2. Upward pass: recursively aggregate multipole moments by moving upward in the tree until level 2 is reached. Denoting by $\mathcal{S}(\mathcal{C})$ the set of children of a given cell $\mathcal{C}$, the transition from a level- $(\ell+1)$ cell to its parent level- $\ell$ cell is based on identities

$$
\begin{aligned}
& \mathcal{R}_{k}^{\mathrm{S}, u}\left(\hat{\boldsymbol{s}} ; \mathcal{C}_{y}^{(\ell)}\right)=\sum_{\mathcal{C}_{y}^{(\ell+1)} \in \mathcal{S}\left(\mathcal{C}_{y}^{(\ell)}\right)} \exp \left[-\mathrm{i} k_{\mathrm{S}} \hat{\boldsymbol{s}} \cdot\left(\boldsymbol{y}_{0}^{(\ell+1)}-\boldsymbol{y}_{0}^{(\ell)}\right)\right] \mathcal{R}_{k}^{\mathrm{S}, u}\left(\hat{\boldsymbol{s}} ; \mathcal{C}_{y}^{(\ell+1)}\right) \\
& \mathcal{R}^{\mathrm{P}, u}\left(\hat{\boldsymbol{s}} ; \mathcal{C}_{y}^{(\ell)}\right)=\sum_{\mathcal{C}_{y}^{(\ell+1)} \in \mathcal{S}\left(\mathcal{C}_{y}^{(\ell)}\right)} \exp \left[-\mathrm{i} k_{\mathrm{P}} \hat{\boldsymbol{s}} \cdot\left(\boldsymbol{y}_{0}^{(\ell+1)}-\boldsymbol{y}_{0}^{(\ell)}\right)\right] \mathcal{R}^{\mathrm{P}, u}\left(\hat{\boldsymbol{s}} ; \mathcal{C}_{y}^{(\ell+1)}\right) .
\end{aligned}
$$

It is essential at this point to emphasize a crucial feature of the elastodynamic multi-level FMM, namely that the number and location of the quadrature points on $S$ are level-dependent (see Section 4.3 for details), a consequence of the previously-mentioned dependence of $L$, the truncation parameter in expansions (13), (14), on the cell size. Hence, application of identities (28a,b) requires an extrapolation procedure furnishing the values of $\mathcal{R}_{k}^{\mathrm{S}, u}, \mathcal{R}^{\mathrm{P}, u}$ at the level- $\ell$ quadrature points from those at the level- $(\ell+1)$ quadrature points (see section 4.4).

3. Transfer: initialize local expansions for each level- $\ell$ cell $\mathcal{C}_{x}^{(\ell)}$ and at each level $2 \leq \ell \leq \bar{\ell}$ using

$$
\begin{aligned}
& \mathcal{L}_{k}^{\mathrm{S}, u}\left(\hat{\boldsymbol{s}}^{(\ell)} ; \mathcal{C}_{x}^{(\ell)}\right)=\sum_{\mathcal{C}_{y}^{(\ell)} \in \mathcal{I}\left(\mathcal{C}_{x}^{(\ell)}\right)} \mathcal{G}_{L}\left(\hat{\boldsymbol{s}}^{(\ell)} ; \boldsymbol{r}_{0} ; k_{\mathrm{S}}\right) \mathcal{R}_{k}^{\mathrm{S}, u}\left(\hat{\boldsymbol{s}}^{(\ell)} ; \mathcal{C}_{y}^{(\ell)}\right) \\
& \mathcal{L}^{\mathrm{P}, u}\left(\hat{\boldsymbol{s}}^{(\ell)} ; \mathcal{C}_{x}^{(\ell)}\right)=\sum_{\mathcal{C}_{y}^{(\ell)} \in \mathcal{I}\left(\mathcal{C}_{x}^{(\ell)}\right)} \mathcal{G}_{L}\left(\hat{\boldsymbol{s}}^{(\ell)} ; \boldsymbol{r}_{0} ; k_{\mathrm{P}}\right) \mathcal{R}^{\mathrm{P}, u}\left(\hat{\boldsymbol{s}}^{(\ell)} ; \mathcal{C}_{y}^{(\ell)}\right)
\end{aligned}
$$

where $\mathcal{I}(\mathcal{C})$, the interaction list of a given cell $\mathcal{C}$ (Fig. 5), is the set of same-level cells which are not adjacent to $\mathcal{C}$ while having a parent cell adjacent to that of $\mathcal{C}$. For a level-2 cell, $(29 \mathrm{a}, \mathrm{b})$ coincides with (24a,b), as $\mathcal{I}\left(\mathcal{C}^{2}\right)$ collects all level-2 cells not adjacent to $\mathcal{C}^{2}$. 
4. Downward pass: for all levels $3 \leq \ell \leq \bar{\ell}$, the local expansion for each level- $\ell$ cell $\mathcal{C}_{x}^{(\ell)}$ is updated with the contribution from the parent level- $(\ell-1)$ cell, by means of the identity

$$
\begin{aligned}
\mathcal{L}_{k}^{\mathrm{S}, u}\left(\hat{\boldsymbol{s}} ; \mathcal{C}_{x}^{(\ell)}\right) & =\mathcal{L}_{k}^{\mathrm{S}, u}\left(\hat{\boldsymbol{s}} ; \mathcal{C}_{x}^{(\ell)}\right)+\exp \left[-\mathrm{i} k_{\mathrm{S}}\left(\hat{\boldsymbol{s}} .\left(\boldsymbol{x}_{0}^{(\ell-1)}-\boldsymbol{x}_{0}^{(\ell)}\right)\right)\right] \mathcal{L}_{k}^{\mathrm{S}, u}\left(\hat{\boldsymbol{s}} ; \mathcal{C}_{x}^{(\ell-1)}\right) \\
\mathcal{L}^{\mathrm{P}, u}\left(\hat{\boldsymbol{s}} ; \mathcal{C}_{x}^{(\ell)}\right) & =\mathcal{L}^{\mathrm{P}, u}\left(\hat{\boldsymbol{s}} ; \mathcal{C}_{x}^{(\ell)}\right)+\exp \left[-\mathrm{i} k_{\mathrm{P}}\left(\hat{\boldsymbol{s}} .\left(\boldsymbol{x}_{0}^{(\ell-1)}-\boldsymbol{x}_{0}^{(\ell)}\right)\right)\right] \mathcal{L}^{\mathrm{P}, u}\left(\hat{\boldsymbol{s}} ; \mathcal{C}_{x}^{(\ell-1)}\right)
\end{aligned}
$$

Similarly to step 2 , application of identity $(30 \mathrm{a}, \mathrm{b})$ requires an inverse extrapolation procedure furnishing the values of $\mathcal{L}_{k}^{\mathrm{S}, u}, \mathcal{L}^{\mathrm{P}, u}$ at the level- $\ell$ quadrature points from those at the level- $(\ell-1)$ quadrature points (see section 4.4).

5. When the leaf level $\ell=\bar{\ell}$ is reached, all local expansions have been computed. The contribution $(\mathcal{K} u)^{\mathrm{FM}}(\boldsymbol{x})$ is evaluated using (26) with the level- $\bar{\ell}$ quadrature points, and the near-field contribution is evaluated according to $(20 \mathrm{a}, \mathrm{b})$ for all level- $\bar{\ell}$ (leaf) cells $\mathcal{C}_{x}$.

The computation of the right-hand side (8) follows the same steps, with the multipole moments $\mathcal{R}_{k}^{\mathrm{S}, u}, \mathcal{R}^{\mathrm{P}, u}$ and local expansions $\mathcal{L}_{k}^{\mathrm{S}, u}, \mathcal{L}^{\mathrm{P}, u}$ replaced with their counterparts $\mathcal{R}_{k}^{\mathrm{S}, t}, \mathcal{R}^{\mathrm{P}, t}$ and $\mathcal{L}_{k}^{\mathrm{S}, t}, \mathcal{L}^{\mathrm{P}, t}$. The above steps are shown in Section 5.2 to have a complexity of at most $O(N \log N)$, with the exception of the direct and inverse extrapolations in steps 2 and 4, whose complexity is $O\left(N^{3 / 2}\right)$.

\subsection{Computation of near-field contributions}

The near-field contributions (20a,b) involve (i) CPV-singular, (ii) weakly-singular and (iii) nonsingular element integrals. CPV-singular integrals are split according to

$$
\begin{aligned}
\text { (P.V.) } \int_{\partial \Omega} u_{i}(\boldsymbol{y}) T_{i}^{k} & (\boldsymbol{x}, \boldsymbol{y} ; \omega) d S_{y} \\
& =\int_{\partial \Omega} u_{i}(\boldsymbol{y})\left[T_{i}^{k}(\boldsymbol{x}, \boldsymbol{y} ; \omega)-T_{i}^{k}(\boldsymbol{x}, \boldsymbol{y})\right] d S_{y}+(\mathrm{P} . \mathrm{V} .) \int_{\partial \Omega} u_{i}(\boldsymbol{y}) T_{i}^{k}(\boldsymbol{x}, \boldsymbol{y}) d S_{y}
\end{aligned}
$$

where $T_{i}^{k}(\boldsymbol{x}, \boldsymbol{y})$ are the traction components of the (singular) static fundamental solution and the difference $T_{i}^{k}(\boldsymbol{x}, \boldsymbol{y} ; \omega)-T_{i}^{k}(\boldsymbol{x}, \boldsymbol{y})$ is non-singular [3]. The remaining CPV integral is then evaluated analytically, taking advantage of the fact that three-noded triangular elements, which have constant unit normal and Jacobian, are used. Weakly-singular integrals (which feature the kernel $U_{i}^{k}(\boldsymbol{x}, \boldsymbol{y} ; \omega)$ ) and non-singular integrals are computed using numerical Gaussian quadrature (the weak singularity being first cancelled by means of a suitable change of coordinates). Finally, when $\partial \Omega$ presents an edge or corner at $\boldsymbol{x}$, the free-term $c_{i j}(\boldsymbol{x})$ is evaluated using the method of [33].

\section{Fast Multipole Method: computational aspects}

Both the single-level and multi-level elastodynamic FMM have been implemented, for three-noded triangular boundary elements, using a public domain version of the GMRES solver [15] with a convergence criterion set to $\|\{\mathcal{K} u-f\}\| /\|\{f\}\| \leq 10^{-3}$. All examples have been run on the same single-processor PC (RAM: 3GB, CPU frequency: $3.40 \mathrm{GHz}$ ). Except where indicated otherwise, the multi-level FMM is used.

The numerical efficiency and accuracy of the FMM is strongly affected by several factors, such as the truncation of the transfer function, the quadrature over the unit sphere and the number of levels, and great care must be taken in the implementation. This section is devoted to a discussion of these issues, and of various algorithmic choices and improvements. The latter are largely based on a transposition to the present elastodynamic context of ideas and methods proposed in $[8,53]$ for the FMM applied to the 3-D frequency-domain Maxwell equations. At several places, illustrative 


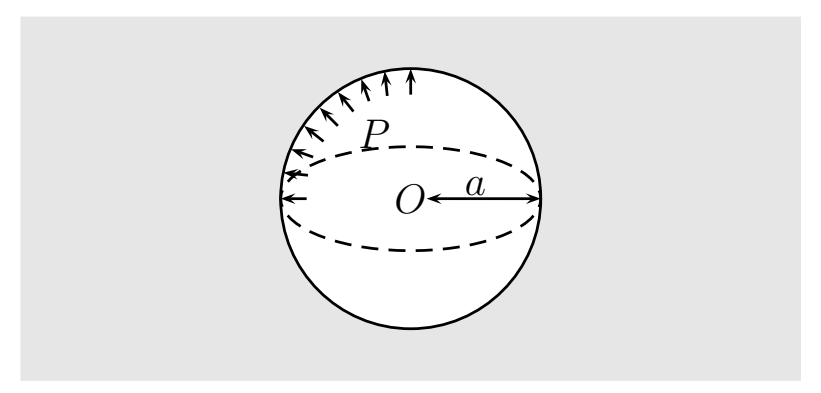

Figure 6: Pressurized spherical cavity: notation.

numerical results for the test problem of a spherical cavity of radius $a$ embedded in an elastic isotropic infinite medium (with $\nu=0.25$ ), subjected to an internal time-harmonic uniform pressure $P$ (Fig. 6) are given. This problem has a simple, spherically-symmetric, exact solution [12], with the radial displacement and stress given in terms of the normalized radial coordinate $\hat{r}=r / a$ by:

$$
\begin{aligned}
u_{r}(\hat{r}) & =\frac{a P}{\mu} \frac{1}{\hat{r}^{2}} \frac{\gamma^{2}\left(1-\mathrm{i} k_{\mathrm{P}} a \hat{r}\right)}{4 \gamma^{2}\left(1-\mathrm{i} k_{\mathrm{P}} a\right)-\left(k_{\mathrm{P}} a\right)^{2}} \exp \left(\mathrm{i} k_{\mathrm{P}} a(\hat{r}-1)\right) \\
\sigma_{r r}(\hat{r}) & =P \frac{1}{\hat{r}^{3}} \frac{\left(k_{\mathrm{P}} a\right)^{2} \hat{r}^{2}-4 \gamma^{2}\left(1-\mathrm{i} k_{\mathrm{P}} a \hat{r}\right)}{4 \gamma^{2}\left(1-\mathrm{i} k_{\mathrm{P}} a\right)-\left(k_{\mathrm{P}} a\right)^{2}} \exp \left(\mathrm{i} k_{\mathrm{P}} a(\hat{r}-1)\right)
\end{aligned}
$$

with the wavenumber $k_{\mathrm{P}}$ and the wave velocity ratio $\gamma$ defined in (3).

\subsection{Truncation of the transfer function}

As already mentioned in section 3.1, the decomposition (10) is shown in [9] to be convergent in the limit $L \rightarrow+\infty$, which immediately implies convergence for the corresponding expressions (13), (14) of the elastodynamic kernels. However, the spherical Hankel functions $h_{p}^{(1)}(z)$ behave like $(p / z)^{p}$ for large $p$ [1] and their evaluation must therefore be avoided for orders $p$ significantly larger than $k\left|\boldsymbol{r}_{0}\right|$. Hence, the truncation level $L$ used in (11) has to be large enough to guarantee sufficient accuracy in (10) while avoiding divergence of the Hankel functions appearing in (11). Appropriate values for $L$ achieving the "numerical convergence" of the transfer function $\mathcal{G}_{L}\left(\tilde{\boldsymbol{s}} ; \boldsymbol{r}_{0} ; k\right)$ are selected using formulae empirically established from numerical experiments. One such formula, known from previous studies on FMMs for Maxwell equations [8], reads:

$$
L(d)=\sqrt{3} k d+C_{\epsilon} \log _{10}(\sqrt{3} k d+\pi) .
$$

In this work, distinct truncation levels $L_{\mathrm{P}}$ and $L_{\mathrm{S}}$ are defined according to (32) with $k=k_{\mathrm{P}}$ and $k=k_{\mathrm{S}}$, respectively. The transfer functions $(15 \mathrm{a}, \mathrm{b})$ and $(16 \mathrm{a}, \mathrm{b})$ are then evaluated using $L=L_{\mathrm{S}}$ and $L=L_{\mathrm{P}}$, respectively. The truncation parameter value defined by (32) is level-dependent through the cell size $d$, and $L$ is (roughly) doubled for each upwards transition to a new level.

Formula (32) features a constant $C_{\epsilon}$ which has to be adjusted from numerical experiments. For that purpose, the test problem is now considered for $N=30726$, with a leaf level $\bar{\ell}=3$ and a leaf-cell size $d^{(\bar{\ell})}=0.6 \lambda_{\mathrm{S}}$ (where $\lambda_{\mathrm{S}}=2 \pi / k_{\mathrm{S}}$ denotes the $S$-wavelength). A subset of 10 columns of the influence matrix $[K]$ are computed using both the present FM-BEM (by performing matrix-vector products $[K]\{u\}$ with all entries of $\{u\}$ set to zero except that corresponding to the selected column of $[K]$, set to unity) and standard BEM techniques. The relative RMS difference between these two sets of matrix columns measures the truncation error introduced by the FMM with finite truncation level $L$. This truncation error, and the CPU time for one FMM iteration, are plotted against $C_{\epsilon}$ in 


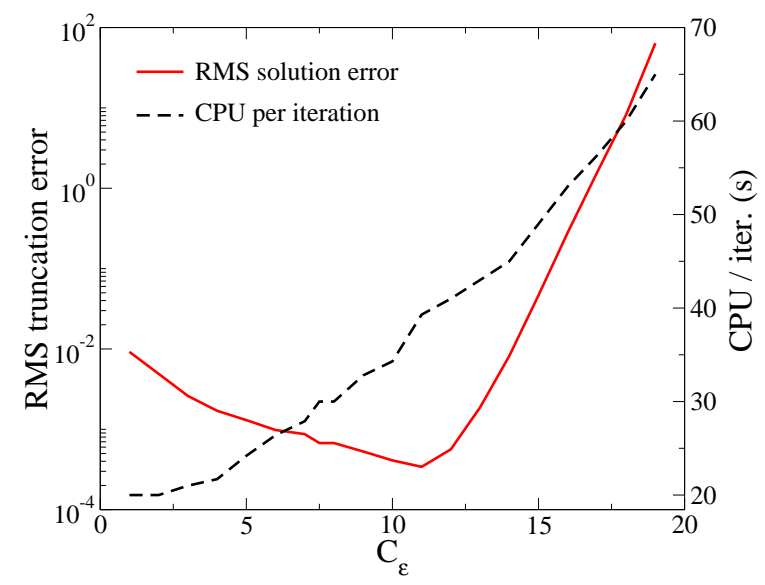

Figure 7: Truncation error and CPU time per iteration as a function of adjustable parameter $C_{\epsilon}$.

Fig. 7. Error levels below $10^{-3}$ are achieved for $5 \leq C_{\epsilon} \leq 12.5$, which corroborates the previouslydiscussed notion of a numerically optimal truncation level $L$. Values of $C_{\epsilon}$ outside the above range lead to values of $L$ that are either too small (insufficient convergence in (10)) or too large (divergence of Hankel functions in (11)). Figure 7 also shows that the CPU time for one FMM iteration increases with $C_{\epsilon}$, which was to be expected since $L$ given by (32) also increases with $C_{\epsilon}$. The value $C_{\epsilon}=7.5$ is found to achieve to keep a good compromise between accuracy and computational cost, and is retained in the present implementation. This observation is consistent with that made in [53] for 3-D electromagnetics.

\subsection{Number of levels}

The choice of the leaf level $\bar{\ell}$ is crucial, as it affects both the overall computational time and the accuracy of the elastodynamic FM-BEM algorithm. A too-small number of levels increases the proportion of near interactions, thus pushing the complexity of the computation closer to $O\left(N^{2}\right)$, while a toolarge number of levels increases the number of transfers between levels (see Table 1 where several values of $\bar{\ell}$ are considered, with $k_{\mathrm{P}} a=6 \pi$ and $N=122886$ ).

The truncation parameter $L$ at any level depends on the leaf-cell size $d^{(\bar{\ell})}$. This is now illustrated with the help of the comparison method and test problem of section 4.1: relative RMS differences between matrices generated by FM-BEM (with $L$ determined at all levels by (32)) and standard BEM produced by this comparison are plotted in Fig. 8 against $C_{\epsilon}$ for several choices of $d^{(\bar{\ell})}$. For small values of $k_{\mathrm{S}} d^{(\bar{\ell})}$, the FM-BEM algorithm is seen to be insufficiently accurate. This stems from the fact that the distances $\left|\boldsymbol{r}_{0}\right|$ between leaf cells scale with $d$, and the spherical Hankel functions in (11) are known to diverge in the small-argument limit. Estimate (17) accordingly predicts that $L$ has a $O(\ln k d)$ divergence in the small cell size limit, and formula (32) does not provide adequate values

\begin{tabular}{|c|c|c|c|}
\hline $\bar{\ell}$ (leaf level) & $k_{\mathrm{S}} d^{(\bar{\ell})} / 2 \pi$ & error / BEM & CPU time / iter (s) \\
\hline 3 & 1.32 & $1.110^{-5}$ & 367 \\
\hline 4 & 0.66 & $4.710^{-4}$ & 134 \\
\hline 5 & 0.33 & $3.710^{-3}$ & 104 \\
\hline 6 & 0.17 & $5.110^{-2}$ & 200 \\
\hline 7 & 0.083 & $1.710^{-1}$ & 380 \\
\hline
\end{tabular}

Table 1: Error and CPU time against the number of levels 


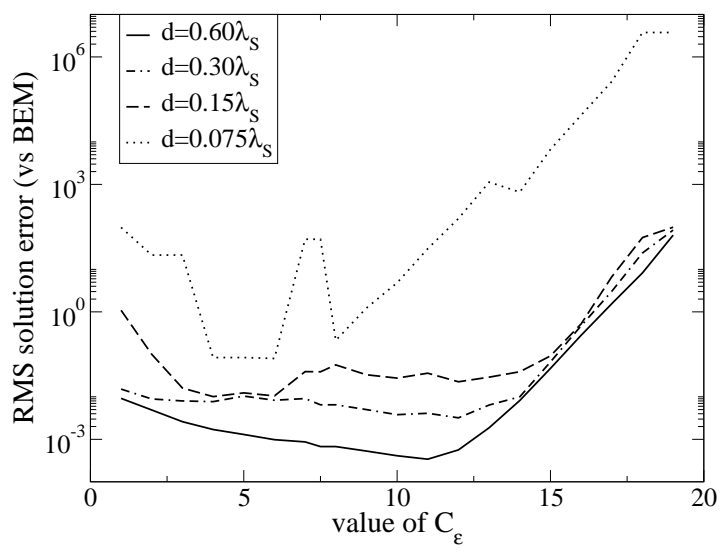

Figure 8: Truncation error as a function of adjustable parameter $C_{\epsilon}$ for several values of leaf-cell size $d^{(\bar{\ell})}$.

of $L$ in this case, even upon increasing the constant $C_{\epsilon}$, as evidenced by the results of Fig. 8. This suggests that the leaf cell size $d^{(\bar{\ell})}$ must be chosen larger to a minimum value $d^{\text {min }}$ to avoid divergence; for instance, results obtained using $d^{(\bar{\ell})}=0.075 \lambda_{\mathrm{S}}$ have very poor accuracy. A minimum leaf cell size $d^{\min }=\lambda / 10$ is adopted in [8]. Accuracy and computational efficiency considerations make higher values of $d^{\mathrm{min}}$ preferable. In this work, the subdivision-stopping criterion defining the leaf level $\bar{\ell}$ is set to: $d^{(\bar{\ell}+1)} \leq d^{\min } \leq d^{(\bar{\ell})}$, with $d^{\min }=0.3 \lambda_{\mathrm{S}}$. Configurations for which cells of size significantly smaller than $d^{\text {min }} \approx 0.3 \lambda_{\mathrm{S}}$ are desirable (e.g. geometries with complex details at sub-wavelength scales) require an adaptation to elastodynamics of approaches combining the diagonal form (10) with other types of expansions valid for low wavenumbers, see [5, 10, 25].

\subsection{Quadrature over the unit sphere}

Another practical issue is the numerical computation of integrals over the unit sphere $S$ in (13), (14). The quadrature method of [8], based on a product rule in the angular spherical coordinates $\theta, \phi$, employs quadrature points and weights of the form $\hat{\boldsymbol{s}}_{q}=\left(\theta_{i}, \phi_{j}\right)$ and $w_{q}=w_{i}^{\theta} w_{j}^{\phi}$, where $\left(\theta_{i}, w_{i}^{\theta}\right)$ $(1 \leq i \leq L)$ correspond to a $L+1$-point Gaussian rule on $[0, \pi]$ while $\left(\phi_{j}, w_{j}^{\phi}\right)$, given by

$$
\phi_{j}=\frac{2 \pi}{2 L+1} j, \quad w_{j}^{\phi}=\frac{2 \pi}{2 L+1} \quad(0 \leq j \leq 2 L),
$$

correspond to a uniform rule on $[0,2 \pi]$. This approach, which employs $Q=(L+1)(2 L+1)$ quadrature points overall, is designed so as to integrate exactly the $L^{2}(S)$-orthonormal set of spherical harmonics $\left(Y_{p, m}(\theta, \phi)\right)_{0 \leq p \leq L,-p \leq m \leq p}$ of order $\leq L$, a requirement which, together with (32), implies that the number of quadrature points must be level-dependent. It is adopted here, in a form slightly modified as explained next.

Reduction of the number of quadrature points. The transfer function $\mathcal{G}_{L}$ given by (11) has the form $\mathcal{G}_{L}\left(\boldsymbol{r}_{0}, \hat{\boldsymbol{s}}, k\right)=\sum_{p=0}^{L} \mathcal{H}_{p}\left(\boldsymbol{r}_{0}\right) P_{p}\left(\cos \left(\hat{\boldsymbol{s}}, \boldsymbol{r}_{0}\right)\right)$. The factor $\mathcal{H}_{p}\left(\boldsymbol{r}_{0}\right)$ does not depend on $\hat{\boldsymbol{s}}$, and is computed once for each $\boldsymbol{r}_{0}$. Then, for each pair $\left(\boldsymbol{r}_{0}, \hat{\boldsymbol{s}}\right)$, the Legendre polynomials are computed by induction:

$$
\left\{\begin{array}{l}
P_{p}(x)=(2-1 / p) x P_{p-1}(x)+(1 / p-1) P_{p-2}(x) \\
P_{0}(x)=1, \quad P_{1}(x)=x
\end{array} \quad\left(x=\frac{\boldsymbol{r}_{0} \cdot \hat{\boldsymbol{s}}}{\left|\boldsymbol{r}_{0}\right||\hat{\boldsymbol{s}}|}\right) .\right.
$$


The Legendre polynomials are known to satisfy the identity $P_{p}(-x)=(-1)^{p} P_{p}(x)$. This can be exploited to reduce the number of quadrature points $\hat{s}$ : a grid that is invariant under the transformation $\hat{s} \rightarrow-\hat{s}$ allows to perform the numerical integration on $S$ with half the original quadrature points. The rule defined by (33) fulfils this invariance provided the uniform rule on $[0,2 \pi]$ is defined in terms of $2 L+2$, rather than $2 L+1$, points. This modified version of (33) features $2(L+1)^{2}$ points, but only $(L+1)^{2}$ points are actually computed, stored and used. As a result, the computing time and memory required by the quadrature are roughly divided by 2 .

\subsection{Extrapolation (direct/inverse)}

The upward translations $(28 \mathrm{a}, \mathrm{b})$ require evaluating multipole moments at level- $\ell$ quadrature points from their values at level- $(\ell+1)$ quadrature points. This important step of the algorithm has a significant impact on the overall CPU time required by the FM-BEM, and hence has to be formulated carefully. A fast method, which takes advantage of the uniform distribution (33) of quadrature points along $\phi$ and exploits $L^{2}(S)$-orthogonality and finite-bandwidth properties of the spherical harmonics, has been proposed in $[8,53]$ and is used here.

With the quadrature points at levels $\ell$ and $\ell+1$ of the form

$$
\begin{aligned}
& \hat{\boldsymbol{s}}_{q}^{(\ell+1)}=\left(\theta_{i}^{(\ell+1)}, \phi_{j}^{(\ell+1)}\right) \quad 0 \leq i \leq L^{(\ell+1)} \quad 0 \leq j \leq 2 L^{(\ell+1)}, \\
& \hat{\boldsymbol{s}}_{q}^{(\ell)}=\left(\theta_{i^{\prime}}^{(\ell)}, \phi_{j^{\prime}}^{(\ell)}\right) \quad 0 \leq i^{\prime} \leq L^{(\ell)} \quad 0 \leq j^{\prime} \leq 2 L^{(\ell)},
\end{aligned}
$$

the values $\mathcal{F}_{i^{\prime} j^{\prime}}=\mathcal{F}\left(\theta_{i^{\prime}}^{(\ell)}, \phi_{j^{\prime}}^{(\ell)}\right)$ at the level- $\ell$ quadrature points of a generic function $\mathcal{F}(\hat{s})=\mathcal{F}(\theta, \phi)$ are extrapolated from those $\mathcal{F}_{i j}=\mathcal{F}\left(\theta_{i}^{(\ell+1)}, \phi_{j}^{(\ell+1)}\right)$ at the level- $(\ell+1)$ quadrature points by means of the following three steps:

$$
\begin{aligned}
& \tilde{\mathcal{F}}_{i m}^{(\ell+1)}=\sum_{j=0}^{2 L^{(\ell+1)}} e^{-\mathrm{i} m \phi_{j}^{(\ell+1)}} \mathcal{F}_{i j}^{(\ell+1)} \quad\left(|m| \leq L^{(\ell+1)}\right) \quad \text { forward Fast Fourier Transform, } \\
& \tilde{\mathcal{F}}_{i^{\prime} m}^{(\ell)}=\sum_{i=0}^{L^{(\ell+1)}} B_{i^{\prime} i}^{m, \ell} \tilde{\mathcal{F}}_{i m}^{(\ell+1)} \quad \text { dense matrix-vector product, } \\
& \mathcal{F}_{i^{\prime} j^{\prime}}^{(\ell)}=\sum_{m=-L^{(\ell+1)}}^{L^{(\ell+1)}} e^{\mathrm{i} m \phi_{j^{\prime}}^{(\ell)}} \tilde{\mathcal{F}}_{i^{\prime} m}^{(\ell)} \quad \text { backward Fast Fourier Transform, }
\end{aligned}
$$

with

$$
B_{i^{\prime} i}^{m, \ell}=\sum_{p=|m|}^{L^{(\ell+1)}} Q_{p}^{m}\left(\cos \theta_{i}^{(\ell+1)}\right) Q_{p}^{m}\left(\cos \theta_{i^{\prime}}^{(\ell)}\right), \quad Q_{p}^{m}(u)=\sqrt{\frac{2 p+1}{4 \pi} \frac{(p-m) !}{(p+m) !}} P_{p}^{m}(u)
$$

Likewise, the downward translations $(30 \mathrm{a}, \mathrm{b})$ require inverse extrapolations from level- $\ell$ quadrature points to level- $(\ell+1)$ points, which are based on a transposed version of the extrapolation:

$$
\begin{array}{rlrl}
\tilde{\mathcal{F}}_{i^{\prime} m}^{(\ell)} & =\sum_{j^{\prime}=0}^{2 L^{(\ell)}} e^{-\mathrm{i} m \phi_{j^{\prime}}^{(\ell)}} \mathcal{F}_{i^{\prime} j^{\prime}}^{(\ell)} & \left(|m| \leq L^{(\ell+1)}\right) & \text { forward Fast Fourier Transform, } \\
\tilde{\mathcal{F}}_{i m}^{(\ell+1)}=\sum_{i^{\prime}=0}^{L^{(\ell+1)}} B_{i^{\prime} i}^{m, \ell} \tilde{\mathcal{F}}_{i^{\prime} m}^{(\ell)} & \text { dense matrix-vector product, } \\
\mathcal{F}_{i^{\prime} j^{\prime}}^{(\ell+1)}=\sum_{m=-L^{(\ell+1)}}^{L^{(\ell+1)}} e^{\mathrm{i} m \phi_{j}^{(\ell+1)}} \tilde{\mathcal{F}}_{i m}^{(\ell+1)} & \text { backward Fast Fourier Transform },
\end{array}
$$


Other extrapolation methods have been proposed [8], some of which being of lower computational complexity but at the cost of further approximation. The above extrapolation method is exact, and will be shown in numerical experiments (section 5) to account for only a modest fraction of the overall CPU time of an elastodynamic FM-BEM analysis, and hence to be satisfactory.

\subsection{Ordering of the transfer operations}

In operations $(29 \mathrm{a}, \mathrm{b})$, the transfer functions $\mathcal{G}_{L}$ need to be evaluated only for vectors $\boldsymbol{r}_{0}$ linking the centers of two same-level cells $\mathcal{C}_{y}$ and $\mathcal{C}_{x}$. Such vectors are integer multiples of the cell size $d$ : $\boldsymbol{r}_{0}=\left(n_{x} n_{y} n_{z}\right) d$. Moreover, at any given level, the transfers are only computed for cells $\mathcal{C}_{y}$ in the interaction list of a given cell $\mathcal{C}_{x}$, i.e. the integers $n_{x}, n_{y}, n_{z}$ necessarily belong to the set $\{-3 \leq$ $\left.n_{x}, n_{y}, n_{z} \leq 3\right\} \backslash\left\{-1 \leq n_{x}, n_{y}, n_{z} \leq 1\right\}$. The maximum number of distinct vectors $\boldsymbol{r}_{0}$ required for performing all operations $(29 \mathrm{a}, \mathrm{b})$ for a given level is therefore $7^{3}-3^{3}=316$. Each transfer matrix can thus be reused many times, especially at the lowest levels. In order to take advantage of this remark, the transfer operations are first sorted according to the vector $\boldsymbol{r}_{0}$. Then, for each $\boldsymbol{r}_{0}$, the transfer matrix is computed using the method of section 4.3. Moreover, the same transfer matrices are used for each GMRES iteration. It is therefore possible to precompute and store on disk each transfer matrix, prior to performing any GMRES iteration.

\subsection{Matrix of near interactions}

The only BEM matrix in the FMM for which storage may be considered is the near-interaction influence matrix $\left[K^{\text {near }}\right]$, such that $\left[K^{\text {near }}\right]\{u\}=\{\mathcal{K} u\}^{\text {near }}$ with reference to (20a), because $\left[K^{\text {near }}\right]$ is sparse. The most common storage strategy for sparse matrices is the Compressed Sparse Row (CSR) approach [46], based on three linear arrays: the nonzero matrix entries (stored row-wise), the column indices, and integer pointers to the beginning of each matrix row in the first two arrays. Products of CSR-stored sparse matrices with vectors are then computed row by row, which prevents one to take advantage of optimized matrix-vector product routines, e.g. those of the BLAS library.

A modification of this storage strategy takes advantage of the structure of the computation of the near interactions, where a cell can interact only with its neighbor cells. The idea is to store blocks representing the interaction of a cell on its neighbor cells (Fig. 9) and then to evaluate matrix-vector products blockwise (instead of termwise). Each block is stored in full-matrix format. For example, the largest model used in the numerical study of complexity of Section 5.3, for which $N=1215291$, features 18351 non-empty leaf cells. The corresponding blockwise-sparse matrix of near interactions is made of 260203 blocks (i.e. a given leaf cell has on average about 14 non-empty adjacent cells, including itself, for this example).

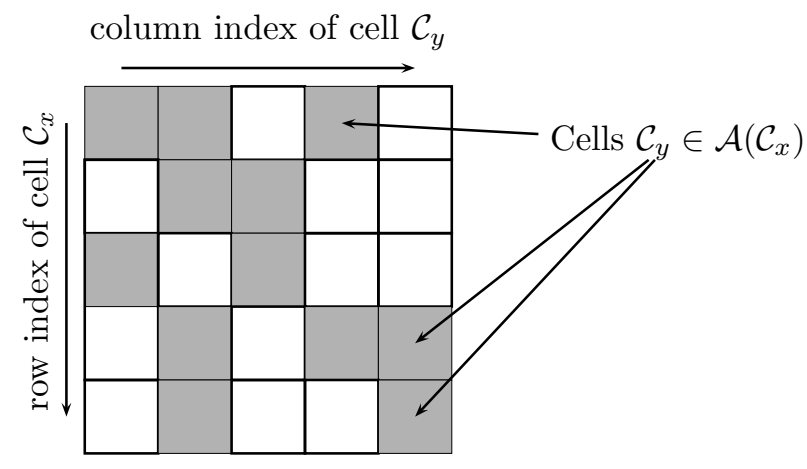

Figure 9: Near interactions matrix (blockwise-sparse storage) 
This storage strategy has two advantages. First, it uses local lists of unknown DOFs for a given cell and its neighbors, instead of the global list. Second, optimized BLAS routines can be used to compute the product of each block of $\left[K^{\text {near }}\right]$ with the corresponding part of the solution vector. Moreover, to reduce the number of blockwise matrix-vector products, only one block is created for each leaf cell $\mathcal{C}_{x}$, with lines and columns corresponding to collocation nodes in $\mathcal{C}_{x}$ and interpolation nodes in all cells $\mathcal{C}_{y} \in \mathcal{A}\left(\mathcal{C}_{x}\right)$, respectively. The matrix entries for each such block are computed by treating the set of elements belonging to all $\mathcal{C}_{y} \in \mathcal{A}\left(\mathcal{C}_{x}\right)$ as a single (small) BEM mesh and using traditional BEM matrix set-up methods.

\subsection{Memory management}

In the multi-level elastodynamic FM-BEM, multipole moments (22a,b) and local expansions (28a,b) are computed for each cell, each level and each quadrature point, and thus arise in large numbers. It is esssential to keep the storage of such quantities to a minimum. The memory needed for a given FM-BEM analysis is affected by the order in which certain tasks are performed. To compute the local expansions $\mathcal{L}_{k}^{\mathrm{S}}, \mathcal{L}^{\mathrm{P}}$ at level $\ell, \mathcal{R}_{k}^{\mathrm{S}}, \mathcal{R}^{\mathrm{P}}$ are needed at level $\ell$ and $\mathcal{L}_{k}^{\mathrm{S}}, \mathcal{L}^{\mathrm{P}}$ at level $(\ell-1)$. One may therefore discard the values of $\mathcal{R}_{k}^{\mathrm{S}}, \mathcal{R}^{\mathrm{P}}$ at level $(\ell+1)$ (and reallocate the corresponding memory) once $\mathcal{R}_{k}^{\mathrm{S}}, \mathcal{R}^{\mathrm{P}}$ are computed at level $\ell$. As schematized in Fig. 10, performing the transfer at level $\ell$ immediately after the upward pass from level $(\ell+1)$ to level $\ell$ allows to restrict the storage to the multipole moments at levels $\ell$ and $(\ell+1)$, and the local expansions at all levels. This ordering hence reduces by about half the memory required for storing multipole moments and local expansions.

Moreover, virtual memory is optimized for large problem sizes, as follows. Multipole moments and local expansions are written on disk (out-of-core). Then, for each step of the multi-level FMM, the needed information is read in the appropriate file and stored back in that file after updating. The maximum virtual memory cost is therefore incurred by the transfer pass at level $\bar{\ell}$, for which all level- $\bar{\ell}$ multipole moments and local expansions must be saved in virtual memory.

For even larger problem sizes, an improved version of this strategy, where the $\ell$-level cells are split into $\mathcal{N}_{\text {gr }}$ groups, has been implemented. The transfer pass is then effected as two nested loops over the $\mathcal{N}_{g r}$ groups, with operations (including the reordering according to vectors $\boldsymbol{r}_{0}$ linking the centers of two same-level cells, see Section 4.5) done only for cells belonging to the two currently active groups. As a result, the virtual memory required by a transfer pass is divided by $\mathcal{N}_{g r}$. This multi-group out-of-core process is applied separately to each pass of the multi-level FMM. In order to define groups of similar size at each level, the number of groups is level- and problem-dependent.

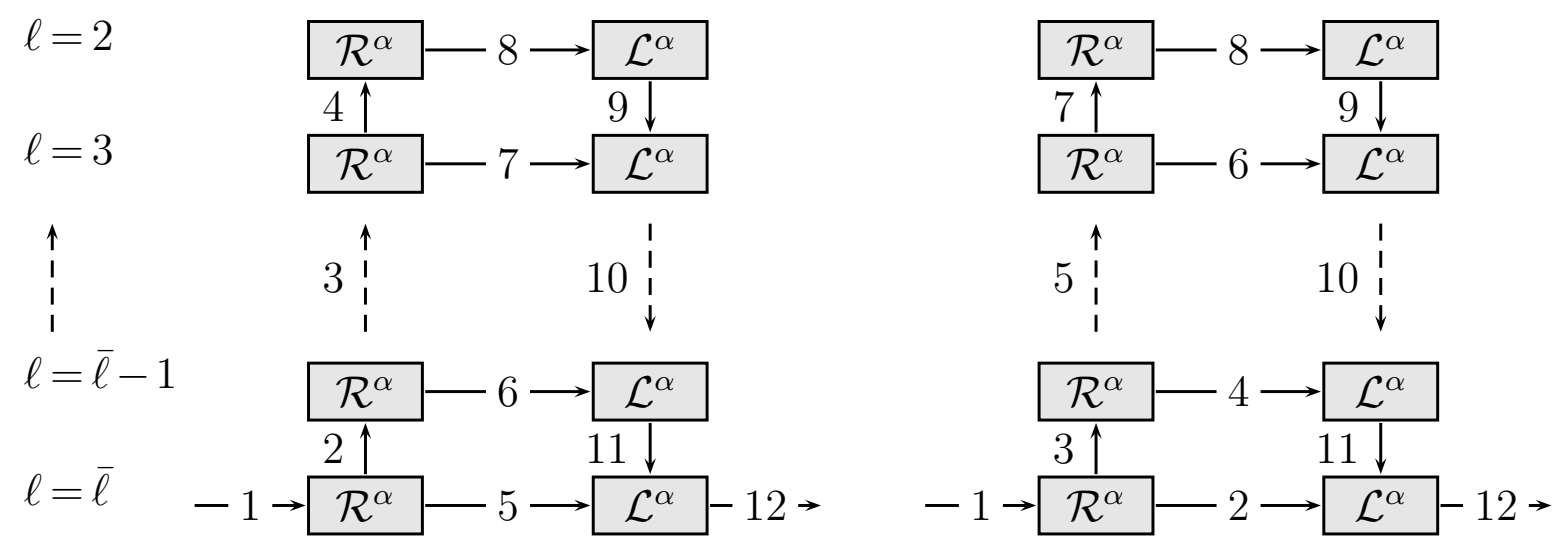

Figure 10: Non-optimal (left) and optimal (right) orderings of the various steps of the multi-level FMM (the numbered arrows indicate the sequential ordering of passes for each case). 


\subsection{Implementation of the elastodynamic FM-BEM: summary}

The elastodynamic multi-level FM-BEM solver implemented in the course of this work, whose features are those discussed in Sections 3 and 4, is summarized for convenience in Figs. 11 and 12.

(a) Octree generation: hierarchically subdivide each cell into 8 children cells, until leaf level $\bar{\ell}$ defined by $d^{\ell \overline{+} 1} \leq d^{\mathrm{min}}=0.3 \lambda_{S} \leq d^{\bar{\ell}}$ is reached

Retain only non-empty children cells

(b) Near contributions:

Compute and store matrix $\left[K^{\text {near }}\right]$ of near interactions (Sec. 4.6)

Compute "near" contribution $\left\{f^{\text {near }}\right\}$, Eq. (20b); store into $\{f\}$

(c) Initial FMM step: preparatory step

Sort vectors $\boldsymbol{r}_{0}=\boldsymbol{y}_{0}-\boldsymbol{x}_{0}$ (Sec. 4.5$)$

Compute and store on disk the transfer matrices

Uses sweep for computing the "far" contribution $\left\{f^{\mathrm{FM}}\right\}$, Eq. (21b); store into $\{f\}$

(d) GMRES initialization:

Set restart parameter to 50, initialize solution vector to $\{u\}=\{0\}$

(e) Generic GMRES iteration; invokes generic FMM step (see Fig. 12)

Invoke (computed and stored in Step (c)) vectors $r_{0}$ and transfer matrices

Use sweep for computing the "far" contribution $\{\mathcal{K} u\}^{\mathrm{FM}}$, Eq. (21a)

Evaluate $\{\mathcal{K} u\}=\{\mathcal{K} u\}^{\mathrm{FM}}+\{\mathcal{K} u\}^{\text {near }}$, Eqs. (19), (20a); pass result to GMRES

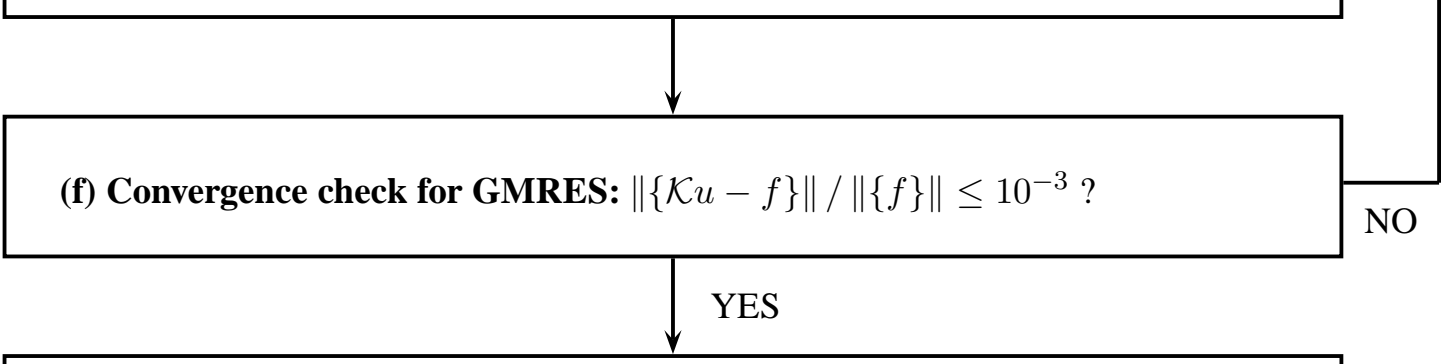

(g) Post-processing of solution:

Evaluate integral representations, create graphics...

Figure 11: Elastodynamic multi-level FM-BEM: schematic description of overall algorithm 
(a) for all leaf cells $\mathcal{C}_{y}^{\bar{\ell}}$

initialization : computation of multipole moments, Eq. (22a)

transfer using $L\left(d^{\ell}\right)=\sqrt{3} k d^{\bar{\ell}}+7.5 \log _{10}\left(\sqrt{3} k d^{\bar{\ell}}+\pi\right)$ terms in expansion, Eqs. $(29 a, b)$

end for

(b) for all levels $\ell=\bar{\ell}-1,2$ (in this order)

for all cells $\mathcal{C}_{y}^{(\ell)}$

for all cells $\mathcal{C}_{y}^{(\ell+1)} \in \mathcal{S}\left(\mathcal{C}_{y}^{(\ell)}\right)$

upward pass, Eqs. $(28 \mathrm{a}, \mathrm{b})$

end for

extrapolation, Eq. (35)

end for

for all cells $\mathcal{C}_{x}^{(\ell)}$

for all cells $\mathcal{C}_{y}^{(\ell)} \in \mathcal{I}\left(\mathcal{C}_{x}^{(\ell)}\right)$

transfer using $L\left(d^{\ell}\right)=\sqrt{3} k d^{\ell}+7.5 \log _{10}\left(\sqrt{3} k d^{\ell}+\pi\right)$ terms in expansion,

Eqs. $(29 a, b)$

end for

end for

end for

(c) for all levels $\ell=3, \bar{\ell}$ (in this order)

for all cells $\mathcal{C}_{x}^{(\ell)}$

downward pass, Eq. (30a,b)

inverse extrapolation, Eq. (36)

end for

end for

(d) for all leaf cells $\mathcal{C}_{x}^{\bar{\ell}}$

for all collocation points $x \in \mathcal{C}_{x}^{\bar{\ell}}$

local expansions Eq. (26)

add "near" part Eq. (20a)

end for

end for

Figure 12: Elastodynamic multi-level FM-BEM: schematic description of generic FMM step

\section{Complexity of the elastodynamic FMM}

In this section, the theoretical complexity of the elastodynamic FMM, i.e. the CPU time spent for each GMRES iteration as a function of $N$, is studied for both the single- and multi-level versions (sections 5.1 and 5.2) and then compared to results from numerical experiments (section 5.3).

\subsection{Theoretical evaluation, single-level FMM}

Noting $d \geq d^{\min }$ the linear cell size, the number of non-empty cells and the number of average DOFs per non-empty cell are $O\left(N / d^{2}\right)$ and $O\left(d^{2}\right)$ respectively; these estimates stem from the fact that the geometrical support of the unknown BE DOFs is two-dimensional. The truncation parameter $L(d)$ given by (32) is such that there is a positive constant $H$ (which depends on $d^{\mathrm{min}}$ ) for which 
$L(d) \leq H d$ for any $d \geq d^{\min }$. Therefore, one may conservatively consider that $L(d)=O(d)$ and, by virtue of (33), that the number $Q$ of quadrature points over $S$ is $Q=O\left(d^{2}\right)$. The main steps of the single-level FMM entail the following computational complexities:

(a) Evaluation of multipole moments $(22 a, b)$ and local expansions (26), for each quadrature point and each cell: $O\left(N d^{2}\right)$

(b) Transfers (24a,b), (25a,b), for each quadrature point and each pair of non-adjacent cells:

$$
O\left(d^{2} \times N / d^{2} \times N / d^{2}\right)=O\left(N^{2} / d^{2}\right) ;
$$

(c) Near interactions (6), for each cell, by means of the product of a $O\left(d^{2}\right) \times O\left(d^{2}\right)$ matrix with a $O\left(d^{2}\right)$ vector: $O\left(N / d^{2} \times d^{4}\right)=O\left(N d^{2}\right)$.

Setting $d=O\left(N^{\alpha}\right)$ the optimal complexity is obtained by minimizing the largest exponent in $N d^{2}=$ $N^{1+2 \alpha}$ and $N^{2} / d^{2}=N^{2-2 \alpha}$. Hence the optimal cell size in the single-level FMM is $d=O\left(N^{1 / 4}\right)$. As a result, the optimal complexity in the single-level FMM in elastodynamics is of order $O\left(N^{3 / 2}\right)$, and is achieved by using $O\left(N^{3 / 4}\right)$ cells.

\subsection{Theoretical evaluation, multi-level FMM}

The leaf cell size $d^{(\bar{\ell})}$ is as small as possible, under the constraint $d^{(\bar{\ell}+1)} \leq d^{\min } \leq d^{(\bar{\ell})}\left(d^{\min }\right.$ being a fixed fraction of $S$ wavelength), as discussed in section 4.2. Assuming a constant number of DOFs per wavelength, $d^{(\bar{\ell})}$ may be considered as independent of $N$ in the complexity analysis. The size $d^{(0)}$ of the largest cells is related to $d^{(\bar{\ell})}$ by $2^{\bar{\ell}} d^{(\bar{\ell})}=d^{(0)}$. Moreover, the fact that the BEM nodes are located on a surface of characteristic diameter $O\left(d^{(0)}\right)$ implies that $d^{(0)}=2^{\bar{\ell}} d^{(\bar{\ell})}=O\left(N^{1 / 2}\right)$. Hence, the total number of levels is:

$$
\bar{\ell}=O(\log N)
$$

and the number of leaf cells is $O(N)$. Moreover, since the DOFs are supported on a surface, each non-empty level- $\ell$ cell has on average 4 non-empty children cells, and therefore holds an average of $N^{(\ell)}=O\left(4^{-\ell} N\right)$ DOFs. The numbers of non-empty cells and of children at each level for the example of a spherical cavity with $N=1215291$ DOFs, shown in Table 2, corroborate this estimate. Lastly, one notes that the level- $\ell$ truncation parameter and the number of level- $\ell$ quadrature points are $L^{(\ell)}=O\left(d^{(\ell)}\right)=O\left(d^{(0)} \times 2^{-\ell}\right)=O\left(N^{1 / 2} \times 2^{-\ell}\right)$ and $Q^{(\ell)}=O\left(\left(d^{(\ell)}\right)^{2}\right)=O\left(N \times 4^{-\ell}\right)$.

Based on the foregoing remarks, the computational complexities associated with the main steps of the multi-level FMM are obtained as:

(i) Multipole moments (22a,b) and local expansions (26), evaluated only at level $\bar{\ell}: O(N)$.

(ii) Transfers (29a,b), performed for each level, each cell $\mathcal{C}_{x}^{(\ell)}$ and each cell $\mathcal{C}_{y}^{(\ell)} \in \mathcal{I}\left(\mathcal{C}_{x}^{(\ell)}\right)$ : $O\left(4^{\ell} \times Q^{(\ell)}\right)=O(N)$ per level, i.e. $O(N \log N)$ overall.

\begin{tabular}{|c|c|c|}
\hline level & number of non-empty cells & number of children \\
\hline 2 & 56 & 4.86 \\
\hline 3 & 272 & 4.26 \\
\hline 4 & 1160 & 4.07 \\
\hline 5 & 4720 & 3.89 \\
\hline 6 & 18351 & - \\
\hline
\end{tabular}

Table 2: Average number of non-empty cells and children at each level 
(iii) Upward and downward passes (28a,b), (30a,b), for each level $\ell$, each cell and each quadrature point $\hat{\boldsymbol{s}}^{(\ell)}: O(N)$ per level, i.e. $O(N \log N)$ overall.

(iv) Direct and inverse extrapolations, for each level $\ell$ and each cell: $O\left(N^{3 / 2}\right)$.

Estimate (ii) relies on the fact that the interaction list of a given cell contains at most $6^{3}-3^{3}=189$ cells, irrespective of the level and the total number of cells. Estimate (iv) stems from the observation that each extrapolation (35) from level $(\ell+1)$ to level $\ell$ (whose total number is $O\left(4^{\ell}\right)$ ) requires $L^{(\ell)}+1$ dense matrix-vector products, each of size $\left(L^{(\ell)}+1\right) \times\left(L^{(\ell+1)}+1\right)$, i.e. $O\left(4^{\ell} \times 2^{-\ell} N^{1 / 2} \times\left(2^{-\ell} N^{1 / 2} \times\right.\right.$ $\left.\left.\left.2^{-(\ell+1)} N^{1 / 2}\right)\right)=O\left(N^{3 / 2} 2^{-(\ell+1}\right)\right)$ operations. Summing these extrapolations from level $\ell=\bar{\ell}$ to $\ell=3$, the obtained cumulative complexity of all extrapolations is $O\left(N^{3 / 2}\right)$ as stated. A similar analysis holds for the cumulative effect of the inverse extrapolation steps (36).

This analysis therefore predicts a theoretical complexity of $O\left(\alpha N \log N+\beta N^{3 / 2}\right)$ per iteration for the multi-level FMM.

\subsection{Numerical study of complexity}

The theoretical complexities just formulated are now compared against recorded CPU times, on the pressurized spherical cavity problem (section 4$)$. This comparison aims in particular at evaluating the respective importances of the $O(\alpha N \log N)$ and $O\left(\beta N^{3 / 2}\right)$ contributions to the overall complexity of the multi-level FMM. Several frequencies are considered, with the size of the BEM models adjusted so as to maintain a mesh density of about 10 nodes per $S$ wavelength (Table 3 ). This complexity study involves problem sizes of up to $N \approx 1.210^{6}$, while the examples of [17] used $N \leq 2.510^{4}$.

\begin{tabular}{|c||cccccccccc|}
\hline$N$ & 30726 & 122769 & 217983 & 389232 & 449835 & 530709 & 635349 & 771912 & 955608 & 1215291 \\
\hline$k_{\mathrm{P}} a / \pi$ & 3.05 & 6.14 & 8.31 & 10.9 & 11.66 & 12.68 & 13.91 & 15.2 & 17.4 & 19.24 \\
\hline
\end{tabular}

Table 3: Numerical study of complexity: BEM model sizes $N$ and non-dimensional frequencies used.

Multi-level FMM: complexity of the main steps. With reference to items (i) to (iv) of section 5.2, the cumulative CPU times recorded for the main steps of the multi-level FMM are compared to the corresponding theoretical complexities for the evaluation of (i) the multipole moments (Fig. 13a) and local expansions (Fig. 13b), (ii) the transfers (Fig. 13c), and (iii-iv) the upward and downward passes including the (direct/inverse) extrapolations (Figs. 13d). For the latter case, coefficients $(\alpha, \beta)$ allowing a best fit of theoretical complexities of the form $O\left(\alpha N \log N+\beta N^{3 / 2}\right)$ to the CPU data are obtained via regression as $(\alpha, \beta)=\left(1.310^{-7}, 9.810^{-9}\right)$ for the upward pass and $(\alpha, \beta)=\left(1.810^{-6}, 8.210^{-8}\right)$ for the downward pass. These values, which are of course code- and computer-dependent, suggest that the importance of the $O\left(N^{3 / 2}\right)$ contribution to the upward and downward passes becomes significant for $N$ above $O\left(10^{5}\right)$.

On Fig. 14 the computation time required by the upward and downward passes and its estimation $\beta N^{3 / 2}$ are compared to the other steps of the algorithm. The results indicate that the $O\left(N^{3 / 2}\right)$ contributions arising from the extrapolations are small compared to the $O(N \log N)$ contributions for BEM model sizes $N=O\left(10^{6}\right)$ or less, for which the extrapolation method of Section 4.4 is therefore satisfactory. Using improved algorithms for extrapolation such as those proposed in [8], of computational complexity lower than $O\left(N^{3 / 2}\right)$, would reduce the elastodynamic FMM complexity to $O(N \log N)$. They may prove essential for BEM models involving several millions DOFs and more.

Overall complexity of the single-level and multi-level FMM. Numerical experiments, in the form of full BEM solutions obtained using the standard BEM, single-level FM-BEM and multi-level FM- 


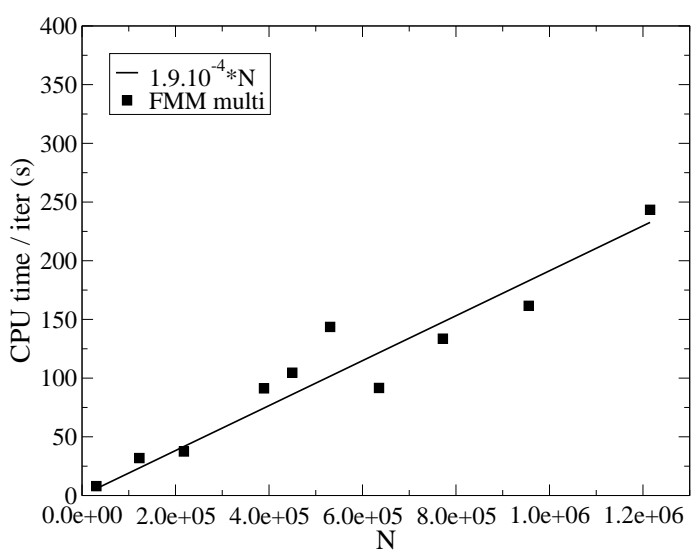

(a) Multipole moments

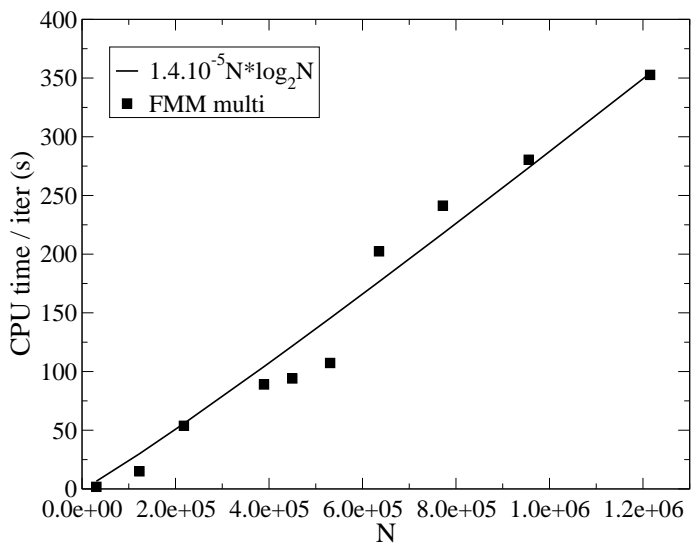

(c) Transfer

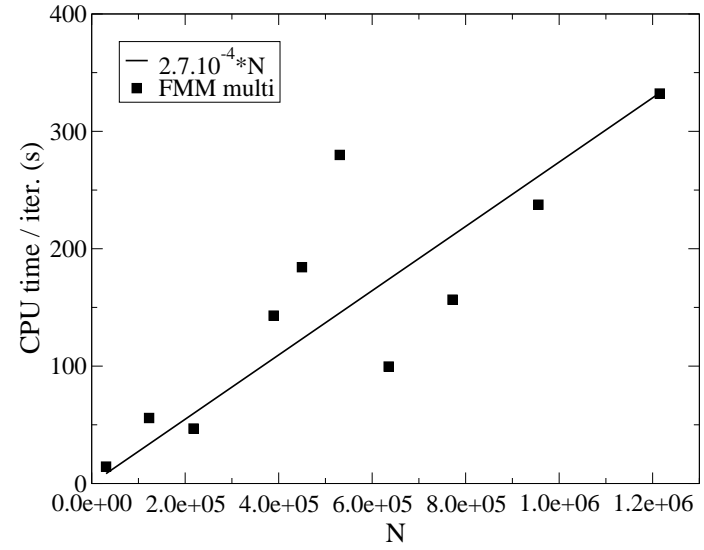

(b) Local expansions

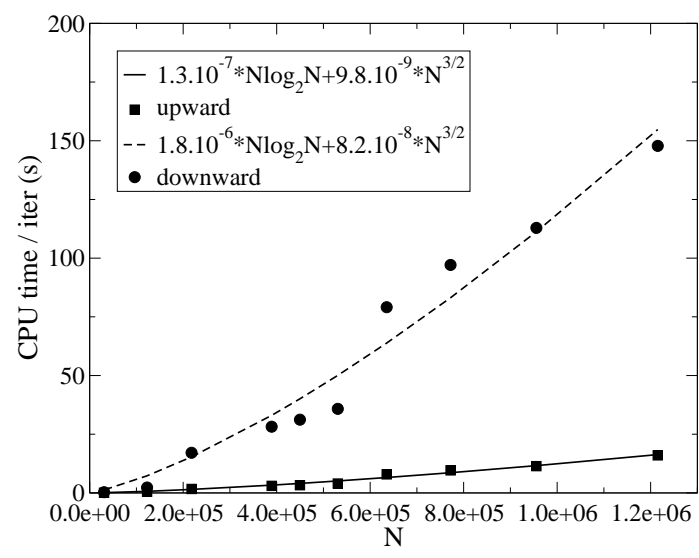

(d) Upward and downward passes

Figure 13: Theoretical complexity and recorded CPU times for the main steps of the multi-level elastodynamic FMM.

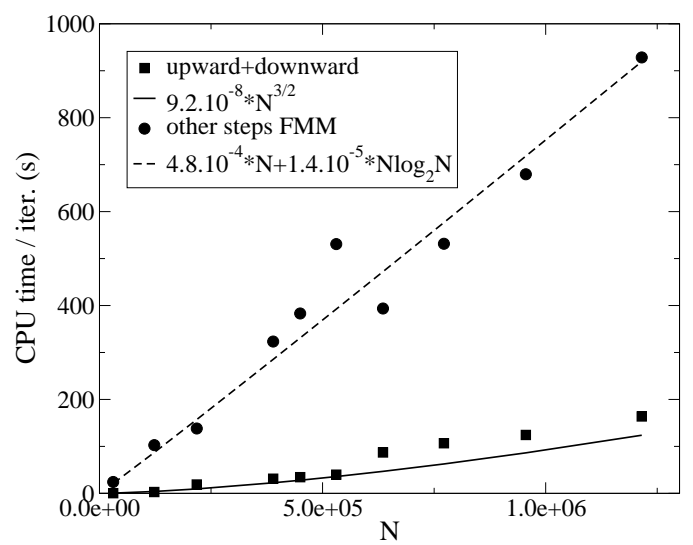

Figure 14: Comparison of the cost of the upward and downward passes to the other steps of the algorithm

BEM on BEM models of respective sizes up to $O\left(10^{4}\right), O\left(10^{5}\right)$ and $O\left(10^{6}\right)$, corroborate the previously discussed theoretical complexities estimates for each approach, as seen in Fig. 15, where the $O\left(N^{3 / 2}\right)$ contribution to the multi-level FMM has been disregarded in accordance with the previous discussion on its effect. 

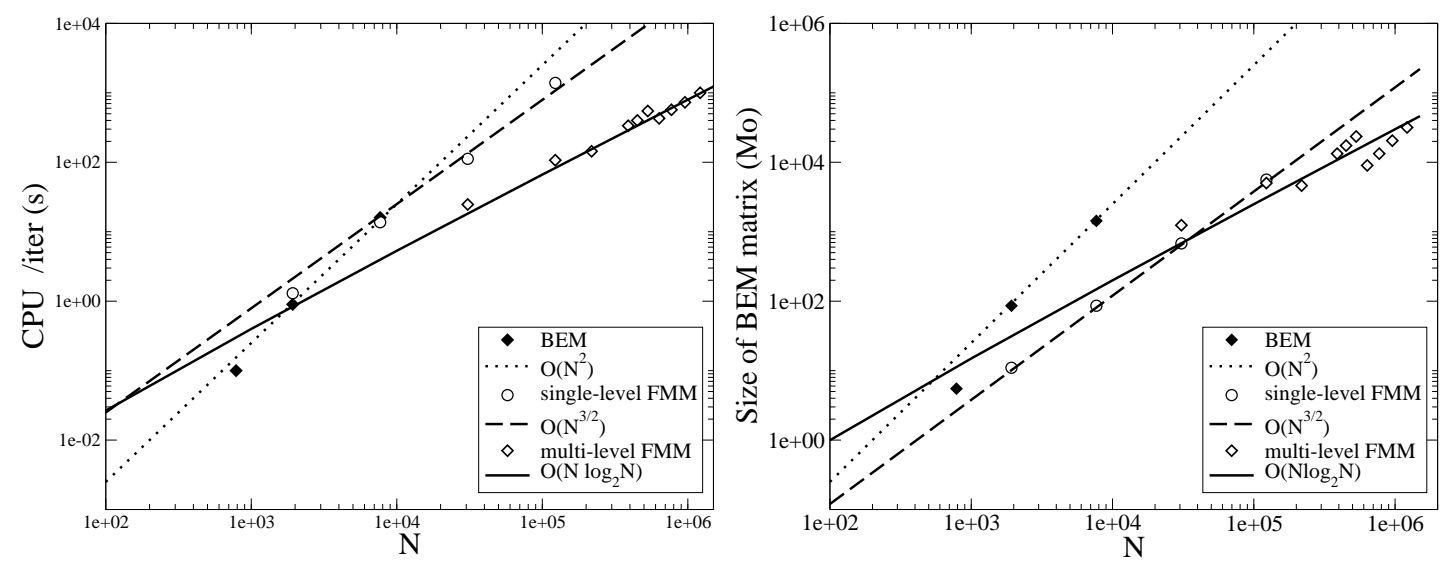

Figure 15: Complexity of the standard BEM, single-level FMM and multi-level FMM (left: CPU time, right: memory)

\subsection{Discussion}

The results of Sections 5.2 and 5.3 are consistent with corresponding studies in $[8,53]$ for electromagnetics, where particular the $O\left(N^{3 / 2}\right)$ complexity of the direct and inverse extrapolations is also pointed out. The $O(N \log N)$ overall complexity is also obtained for the method stable at all frequencies proposed in [10]. In contrast, the elastodynamic FM-BEM of [17] uses a level-independent value for the truncation parameter $L$. This variant avoids the need for direct and inverse extrapolation but requires $L=O\left(k_{\mathrm{S}} d^{(0)}\right)=O\left(N^{1 / 2}\right)$ by virtue of (32). Revisiting steps (i), (ii) and (iii) of Section 5.2 with fixed values for $L=O\left(N^{1 / 2}\right)$ and $Q=O\left(L^{2}\right)=O(N)$, one finds a $O\left(N^{2}\right)$ complexity for that approach, as remarked also in [37]. In comparison, static FM-BEMs for static problems are known to have $O(N)$ complexity [30,37] since the truncation parameter in the multipole expansion in that case depends neither on the level nor on the problem size.

\section{Numerical examples}

First, additional numerical results for the example of a pressurized spherical cavity, introduced in Section 4, are presented. Then, the more complex example of the diffraction of an incident $\mathrm{P}$ plane wave by a spherical cavity, for which an exact solution is also available, further demonstrates the good accuracy of the present FMM. Finally, the usefulness of the proposed FMM formulation is illustrated on the scattering of a seismic plane $\mathrm{P}$ wave by an irregular half-space model. For all results presented therein, the following computational parameters were used: $C_{\epsilon}=7.5, d^{\mathrm{min}}=0.3 \lambda_{\mathrm{S}}$ (unless indicated otherwise), and a convergence threshold defined by $\|\{f-\mathcal{K} u\}\| /\|\{f\}\| \leq 10^{-3}$ (using the notations of equation (9)) for GMRES.

\subsection{Pressurized spherical cavity}

The example configuration defined in Section 4 is again used. First, numerically-computed solutions are compared for four non-dimensional frequencies to the corresponding exact solution (31). The stopping criterion relative to cell subdivision proposed in Section 4.2 led to four levels for the highest frequency considered $\left(k_{\mathrm{P}} a / \pi=2\right)$. Four levels were also used for the other three results in order to ensure that a sufficient proportion of the computations utilize multipole expansions (the subdivisionstopping criterion being hence disregarded for these cases). For each frequency, relative RMS errors for the radial displacement on the cavity wall and over the radial interval $a<r \leq 3 a$ are presented in 


\begin{tabular}{|c|c|c|c|c|}
\hline$k_{\mathrm{P}} a / \pi$ & 0.1 & 0.50 & 1.00 & 2.00 \\
\hline$\#$ nodes $/ \lambda_{\mathrm{S}}$ & 80 & 16 & 8 & 4 \\
\hline RMS error, $r=a$ (cavity wall) & 0.025 & 0.006 & 0.006 & 0.021 \\
\hline RMS error, $a<r \leq 3 a$ (domain) & 0.011 & 0.006 & 0.008 & 0.031 \\
\hline
\end{tabular}

Table 4: Pressurized spherical cavity: RMS solution error on the cavity and in the domain

\begin{tabular}{|c|r|c|r|}
\hline \# nodes per S-wavelength & $N$ & RMS solution error on cavity & CPU time per iter.(s) \\
\hline 2.5 & 1926 & $2.010^{-2}$ & 1.5 \\
\hline 5 & 7686 & $4.610^{-3}$ & 3.7 \\
\hline 10 & 30726 & $1.310^{-3}$ & 14.2 \\
\hline 20 & 122886 & $4.010^{-4}$ & 85.1 \\
\hline
\end{tabular}

Table 5: Pressurized spherical cavity: influence of the number of nodes per S-wavelength on the RMS solution error and the CPU time per iteration.

Table 4. The present FM-BEM is seen to be quite accurate, even in the low-frequency case $\left(k_{\mathrm{P}} a / \pi=\right.$ 0.1 ) for which the accuracy of FMM expansions of the form (10) is known to deteriorate [8], whereas the standard BEM does not [7].

Next, the effect of the number of nodes per S-wavelength on solution accuracy is examined. For that purpose, the cavity radius $a$ and angular frequency $\omega$ are kept constant (with $k_{\mathrm{P}} a=3 \pi$ ), while four BEM meshes with increasing mesh densities are used. The corresponding numbers of nodes per S-wavelength are given in Table 5 (first column). The relative solution errors observed for these meshes (Table 5, second column) indicate that a good solution accuracy requires a minimum of 5 nodes per S-wavelength. The corresponding observed CPU times per iteration (Table 5, third column) increase due to the combined effect of mesh refinement and truncation parameter (32). The numerical results presented in the remainder of this article have been obtained using meshes featuring a minimum of 10 nodes per S-wavelength.

\subsection{Diffraction of an incident plane $P$ wave by a spherical cavity}

The geometrical configuration and material parameters are as in the previous example, but the cavity surface is now traction-free. An incident plane $\mathrm{P}$-wave propagates along the positive $z$-direction (Fig. 16). Two frequencies are considered, defined by $k_{\mathrm{P}} a / \pi=1$ and $k_{\mathrm{P}} a / \pi=4$, with respective problem sizes $N=7686$ and $N=122886$. The numerical results are compared to the analytical solution given in [12] (which, incidentally, features a typographical error corrected in [7]).
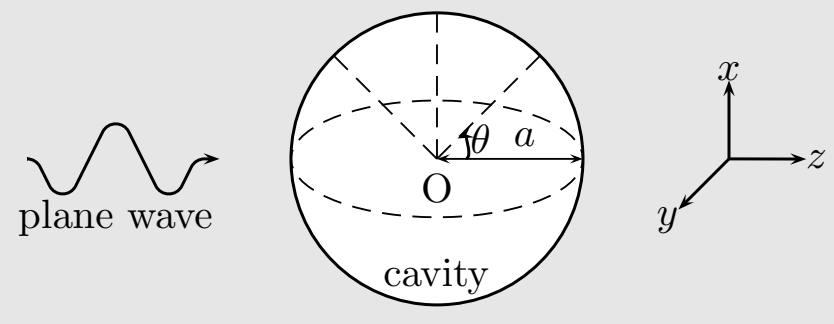

Figure 16: Diffraction of an incident plane $P$ wave by a spherical cavity: notation 
$\theta=0$
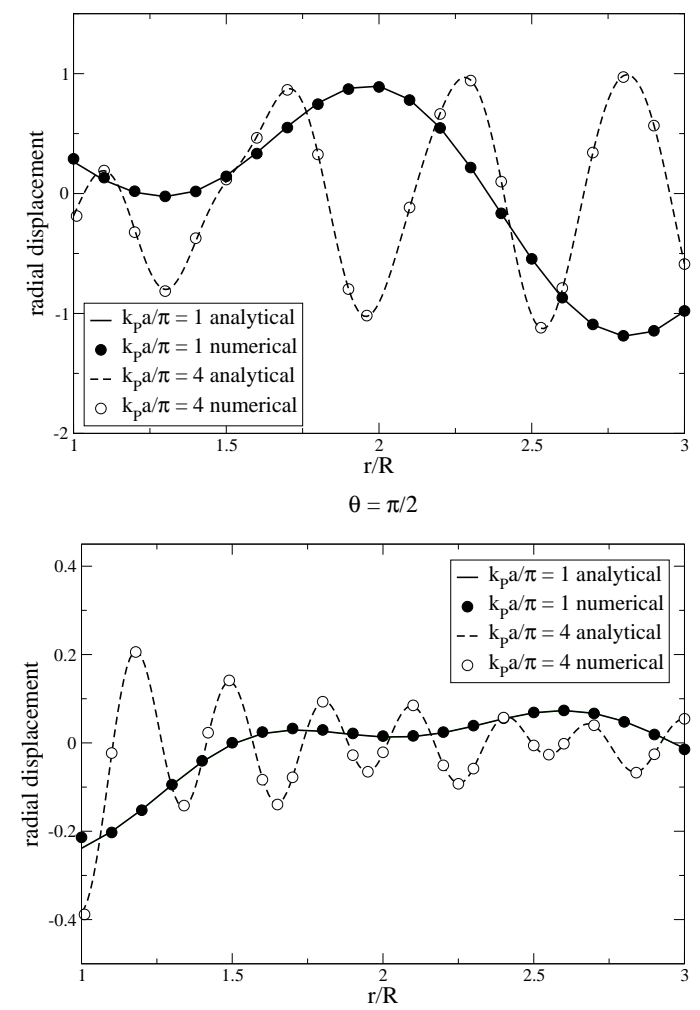

$\theta=\pi / 4$
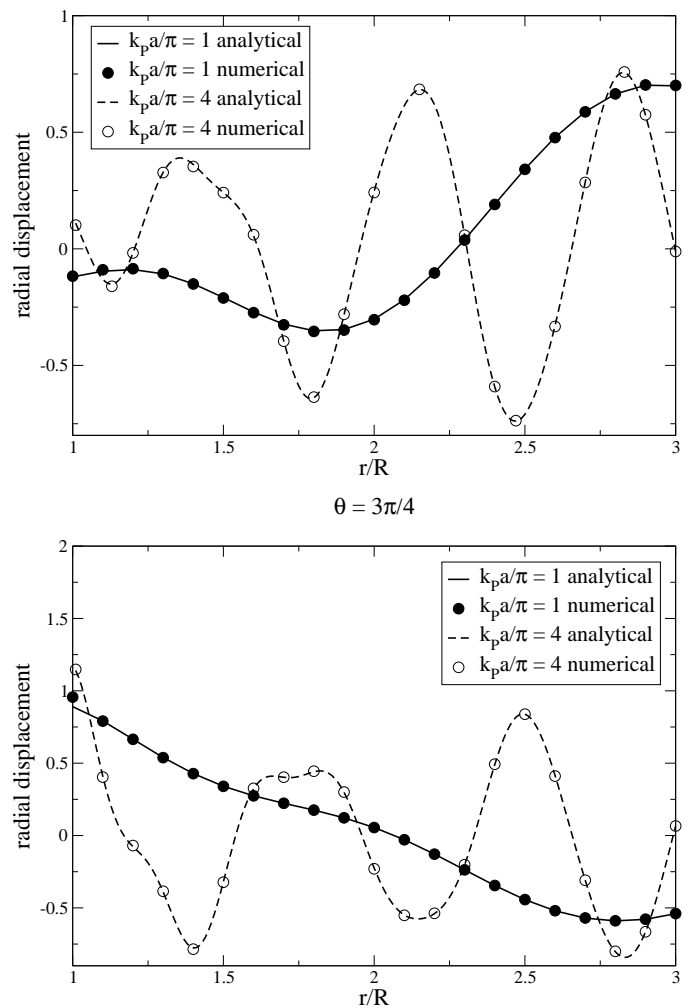

Figure 17: Diffraction of an incident plane $P$ wave by a spherical cavity: comparison of the numerical FMM and analytical solutions for normalized frequencies $k_{P} a / \pi=1,4$ and azimuths $\theta=0, \pi / 4, \pi / 2,3 \pi / 4$.

The numerical results are computed along radial straight lines emanating from the cavity center in directions $(\theta=0, \pi / 4, \pi / 2,3 \pi / 4)$ in the $x$ - $z$ plane. Figure 17 shows the real part of the radial displacement against the normalized radial coordinate $r / a$. The subdivision-stopping criterion employed for cases $k_{\mathrm{P}} a / \pi=1$ and $k_{\mathrm{P}} a / \pi=4$ corresponds respectively to $d^{\mathrm{min}}=0.2 \lambda_{\mathrm{S}}$ and $d^{\mathrm{min}}=0.3 \lambda_{\mathrm{S}}$. The numerical results obtained using the present FM-BEM are seen to agree very well with the exact solution for the two frequencies considered, even along the $\theta=\pi / 2$ direction corresponding to grazing incidence. For the case $k_{\mathrm{P}} a / \pi=4$, a solution CPU time of $44 \mathrm{~s}$ per iteration (144 GMRES iterations, no preconditioning) is recorded. In Table 6 , the influence of the choice of leaf cell size (see Sec-

\begin{tabular}{|ll|r|cccc|}
\hline & & $d^{\min }$ & $\theta=0$ & $\theta=\pi / 4$ & $\theta=\pi / 2$ & $\theta=3 \pi / 4$ \\
\hline$k_{\mathrm{P}} a / \pi=1$ & $(N=7686)$ & $0.2 \lambda_{\mathrm{S}}$ & $9.210^{-3}$ & $2.610^{-3}$ & $2.210^{-2}$ & $8.610^{-4}$ \\
& & $0.1 \lambda_{\mathrm{S}}$ & $9.610^{-3}$ & $8.610^{-3}$ & $9.210^{-3}$ & $4.910^{-3}$ \\
& & $0.05 \lambda_{\mathrm{S}}$ & $1.110^{-2}$ & $2.310^{-2}$ & $4.810^{-2}$ & $2.110^{-2}$ \\
& & $0.02 \lambda_{\mathrm{S}}$ & $4.210^{-2}$ & $3.110^{-2}$ & $3.110^{-1}$ & $8.510^{-2}$ \\
\hline$k_{\mathrm{P} a} a=4$ & $(N=122886)$ & $0.3 \lambda_{\mathrm{S}}$ & $1.410^{-2}$ & $4.410^{-3}$ & $2.310^{-2}$ & $5.610^{-3}$ \\
& & $0.2 \lambda_{\mathrm{S}}$ & $1.410^{-2}$ & $4.210^{-3}$ & $2.010^{-2}$ & $5.210^{-3}$ \\
& & $0.1 \lambda_{\mathrm{S}}$ & $1.710^{-2}$ & $1.510^{-2}$ & $4.610^{-2}$ & $6.810^{-3}$ \\
& & $0.05 \lambda_{\mathrm{S}}$ & $1.410^{-1}$ & $6.810^{-2}$ & $2.610^{-1}$ & $4.610^{-2}$ \\
& & $0.02 \lambda_{\mathrm{S}}$ & $5.810^{-1}$ & $3.510^{-1}$ & $6.010^{-1}$ & $2.110^{-1}$ \\
\hline
\end{tabular}

Table 6: Diffraction of an incident plane $P$ wave by a spherical cavity: influence of leaf cell size on solution error. 
tion 4.2) is further examined. Results obtained by choosing $d^{\min } \geq 0.1 \lambda_{\mathrm{S}}$ are satisfactorily accurate. On the other hand, solution errors are seen to deteriorate markedly whenever values $d^{\text {min }}<0.1 \lambda_{S}$ are used. These results corroborate the validity of the recommended value $d^{\min } \geq 0.3 \lambda_{\mathrm{S}}$ proposed in Section 4.2 on the basis of an essentially one-dimensional test problem. Some of the values of $d^{\min }$ smaller than $0.3 \lambda_{\mathrm{S}}$ also lead to acceptable solution errors for this example. This however cannot be expected to be always true, as the test of Section 4.2 indicates.

\subsection{Diffraction of an incident $P$ plane wave by a semi-ellipsoidal canyon}

This example is concerned with the diffraction by a semi-ellipsoidal canyon of a plane P-wave of unit amplitude travelling in an elastic homogeneous irregular half-space (Fig. 18). A right-handed Cartesian frame $(x, y, z)$ is defined so that the elastic half-space occupies the region $\{(x, y, z) \mid z \geq$ $0\}$. The surface of the canyon is ellipsoidal, with semiaxes $b, a, a$ respectively aligned along the coordinate directions $x, y, z$. The plane wave travels along direction $\sin \theta_{0} \boldsymbol{e}_{y}-\cos \theta_{0} \boldsymbol{e}_{z}$. The semiellipsoidal surface of the canyon and the surrounding portion of free surface lying inside a disk of radius $D>a, b$ are discretized using boundary elements. Such a configuration is representative of a "topographic site effect" in seismology and has been the subject of numerous studies, see [13, 28, 29, 57] and [7, 26, 34, 40,49] where diffraction of waves by surface heterogeneities is considered.

Semi-spherical canyon and vertical incident P-wave. First, the diffraction of a vertical incident plane P-wave by a semi-spherical canyon is considered (i.e. $b=a$, see Fig. 18), with $\nu=0.25$. Results obtained by the present FM-BEM for the (low) normalized frequency $k_{\mathrm{P}} a=0.25 \pi$, by means of a BE mesh featuring $N=23382$ DOFs, are compared to corresponding results from [49] (based on a semi-analytical approach) and [40] (obtained using a standard elastodynamic BEM). In this case, the subdivision-stopping threshold used is $d^{\mathrm{min}}=0.15 \lambda_{\mathrm{S}}$, resulting in a leaf level $\bar{\ell}=3$. Figure 19
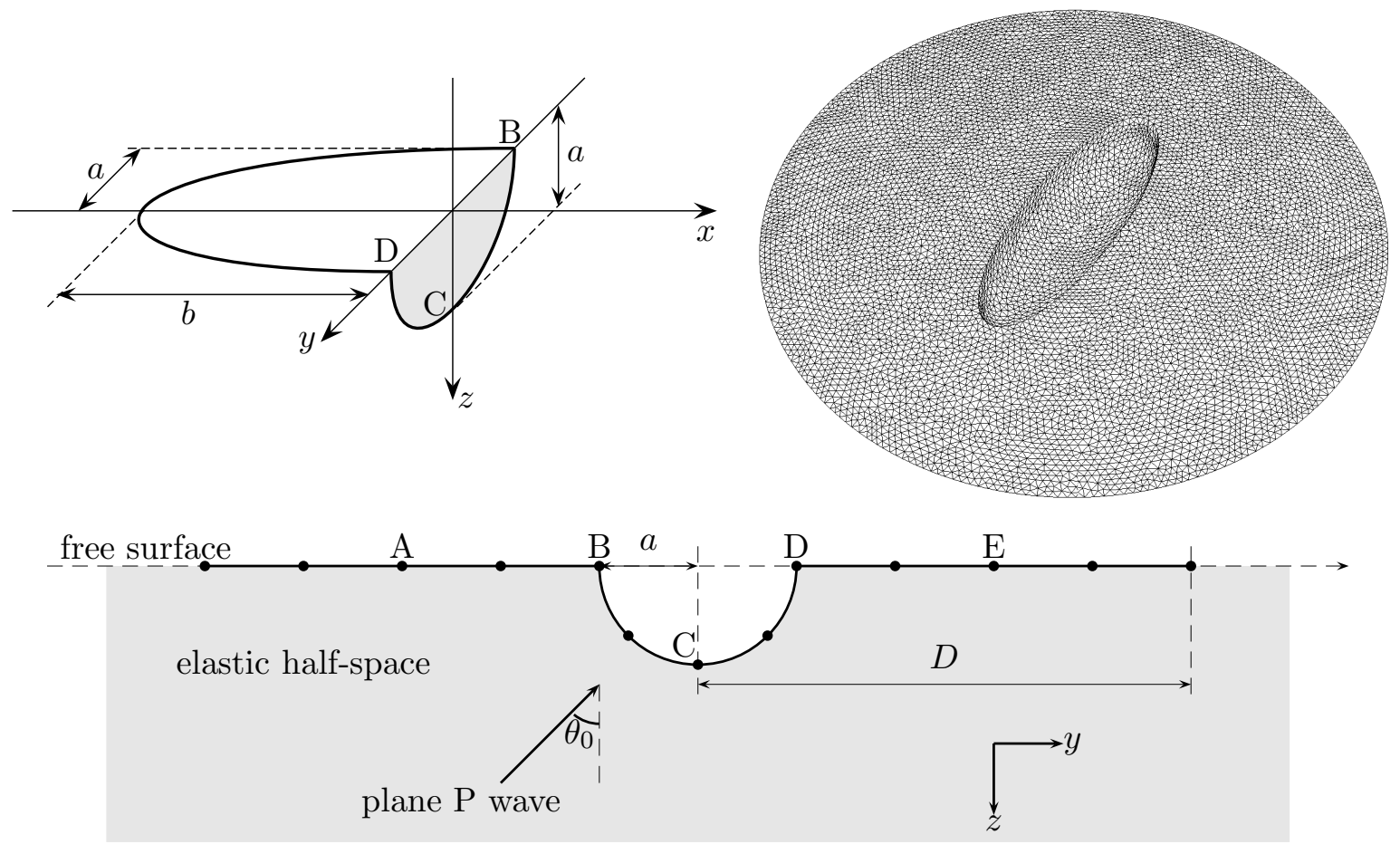

Figure 18: Diffraction of an oblique incident $P$ plane wave by a semi-ellipsoidal canyon: notation (top left and bottom); sample BEM mesh, with $N=25788$ (top right). 


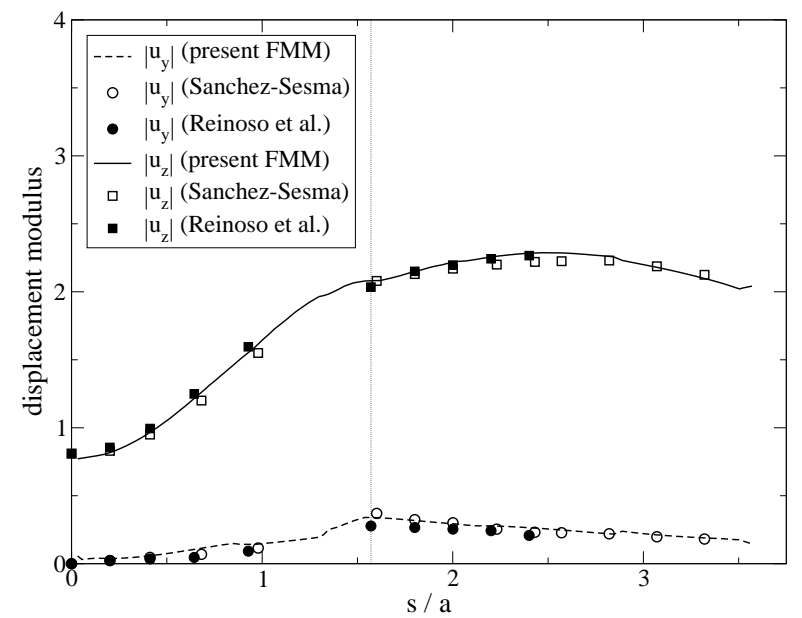

Figure 19: Diffraction of an incident $P$ plane wave by a semi-spherical canyon: horizontal and vertical computed displacement on line CDE (with points $C, D, E$ defined on Fig. 18) plotted against normalized arc-length coordinate s/a along CDE (normalized frequency $k_{P} a / \pi=0.25$ ). Comparison of present FMM solution to results from Sánchez-Sesma [49] and Reinoso et al. [40]

shows that the horizontal and vertical displacements along line CDE (with points C, D, E defined in Fig. 18) produced by the three approaches are in good agreement. Note that the corresponding results in $[40,49]$ are plotted against the horizontal coordinate $y$, whereas the arc-length coordinate $s$ along $\mathrm{ABC}$ is used in Fig. 19. The same value $D=3 a$ of the truncation radius has been used for all three sets of results. The present computation required 7 GMRES iterations and 6 s of CPU time per iteration.

Moreover, the FM-BEM allows to deal with non-dimensional frequencies significantly higher than those considered in previous studies. Figure 20 shows the displacements along line ABC computed for a nondimensional frequency $k_{\mathrm{P}} a / \pi=5$ using the present method. This time, the problem size $N=287946$ is well beyond the capabilities of standard BEM. This computation, performed with a leaf level $\bar{\ell}=6$, required 86 GMRES iterations (without preconditioning) and $163 \mathrm{~s}$ of CPU time per iteration. The displacement near the canyon edge (i.e. $y=a$ and $s=\pi a / 2$, see Fig. 18) has strong variations, as expected.

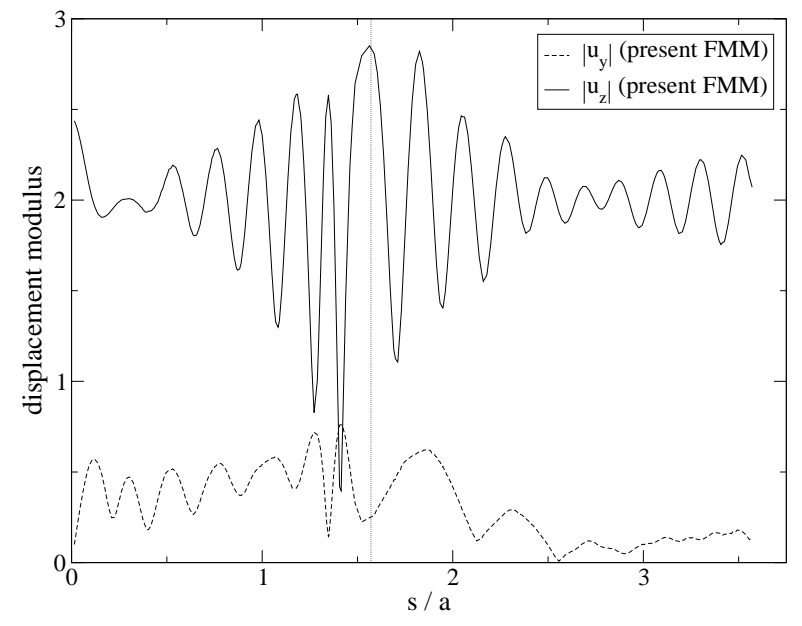

Figure 20: Diffraction of an incident $P$ plane wave by a semi-spherical canyon: horizontal and vertical computed displacement on line CDE (with points $C, D, E$ defined on Fig. 18) plotted against normalized arc-length coordinate s/a along CDE (normalized frequency $k_{P} a / \pi=5$ ) 


\begin{tabular}{|l|r|r|r|r|r|l|}
\hline & $k_{\mathrm{P}} a / \pi=0.25$ & $k_{\mathrm{P}} a / \pi=0.5$ & $k_{\mathrm{P}} a / \pi=0.75$ & $k_{\mathrm{P}} a / \pi=1.5$ & $k_{\mathrm{P}} a / \pi=5$ & $k_{\mathrm{P}} a / \pi=10$ \\
\hline$D=3 a$ & $7(23382)$ & $10(23382)$ & $12(23382)$ & $19(23382)$ & $86(287946)$ & $>280(1145700)$ \\
\hline$D=5 a$ & $7(61875)$ & $10(61875)$ & $15(61875)$ & $28(61875)$ & $159(774180)$ & \\
\hline$D=7 a$ & $8(77565)$ & $13(77565)$ & $17(77565)$ & $43(77565)$ & & \\
\hline$D=20 a$ & $14(98844)$ & $39(98844)$ & $43(98844)$ & & & \\
\hline
\end{tabular}

Table 7: Diffraction of a plane wave by a semi-spherical canyon: number of GMRES iterations for various truncation radii $D$ and nondimensional frequencies $k_{P} a / \pi$ with, in parentheses, the corresponding problem sizes $N$.

The size of the problems that can be solved is now limited by the number of iterations of the iterative solver. The number of iterations required for convergence of the GMRES solver, reported in Table 7 for various problem sizes $N$ and (non-dimensional) frequencies $k_{\mathrm{P}} a / \pi$, clearly depend on both $N$ and $k_{\mathrm{P}} a / \pi$. Reducing the iteration count requires a preconditioning strategy. This critical component of the development of efficient FM-BEM algorithms remains in the authors' view a largely open issue and is not addressed here. In [17], a block diagonal matrix is used. Other strategies for defining preconditioning matrices, found to be effective in the context of electromagnetic FM-BEMs, include performing an incomplete LU decomposition of $[K][52]$ or using the SParse Approximate Inverse [2]. A comparative performance study of available preconditioning strategies remains to be done for the elastodynamic FM-BEM.

Semi-ellipsoidal canyon and oblique incident P-wave. Finally, a fully three-dimensional configuration is considered, namely the scattering of an oblique incident $\mathrm{P}$-wave by a semi-ellipsoidal canyon (with $b=3 a$ and $\theta_{0}=\pi / 6$, see Fig. 18), with $\nu=1 / 3$. This problem has been previously studied in [13] by means of a wave function expansion and, for low frequencies, in [40] using a standard BEM. Results obtained by the present FM-BEM for the (low) normalized frequency $k_{\mathrm{S}} a / \pi=0.5$, by means of a BE mesh featuring $N=25788$ DOFs shown in Fig. 18, are compared to corresponding numerical results from [40]. Figure 21 shows that the horizontal and vertical displacements produced by both approaches, plotted against the normalized arc-length coordinate $s / a$ along line ABCDE (with

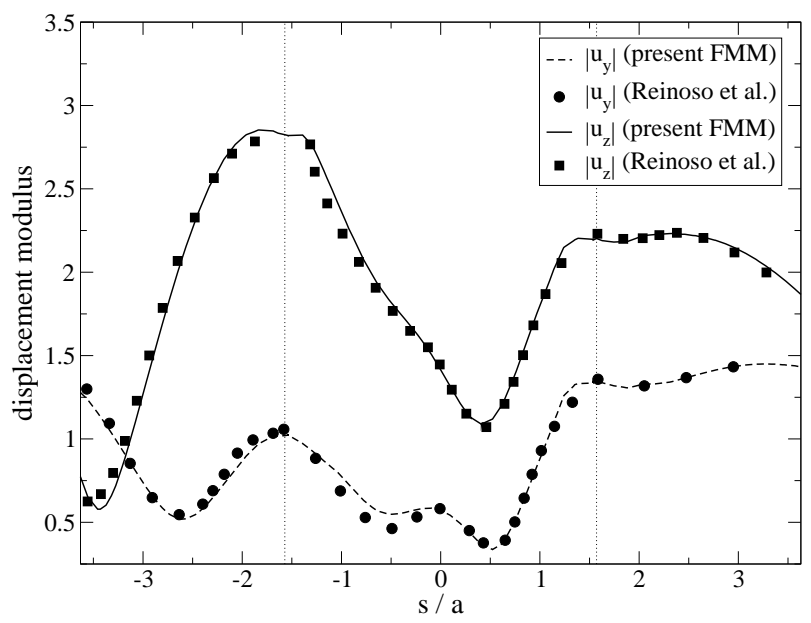

Figure 21: Diffraction of an oblique incident $P$ plane wave by a semi-ellipsoidal canyon: horizontal and vertical computed displacement on line $A B C D E$ (with points $A, B, C D, E$ defined on Fig. 18) plotted against normalized arc-length coordinate s/a along ABCDE (normalized frequency $\left.k_{S} a / \pi=0.5\right)$. Comparison of present FMM solution to results from Reinoso et al. [40] 

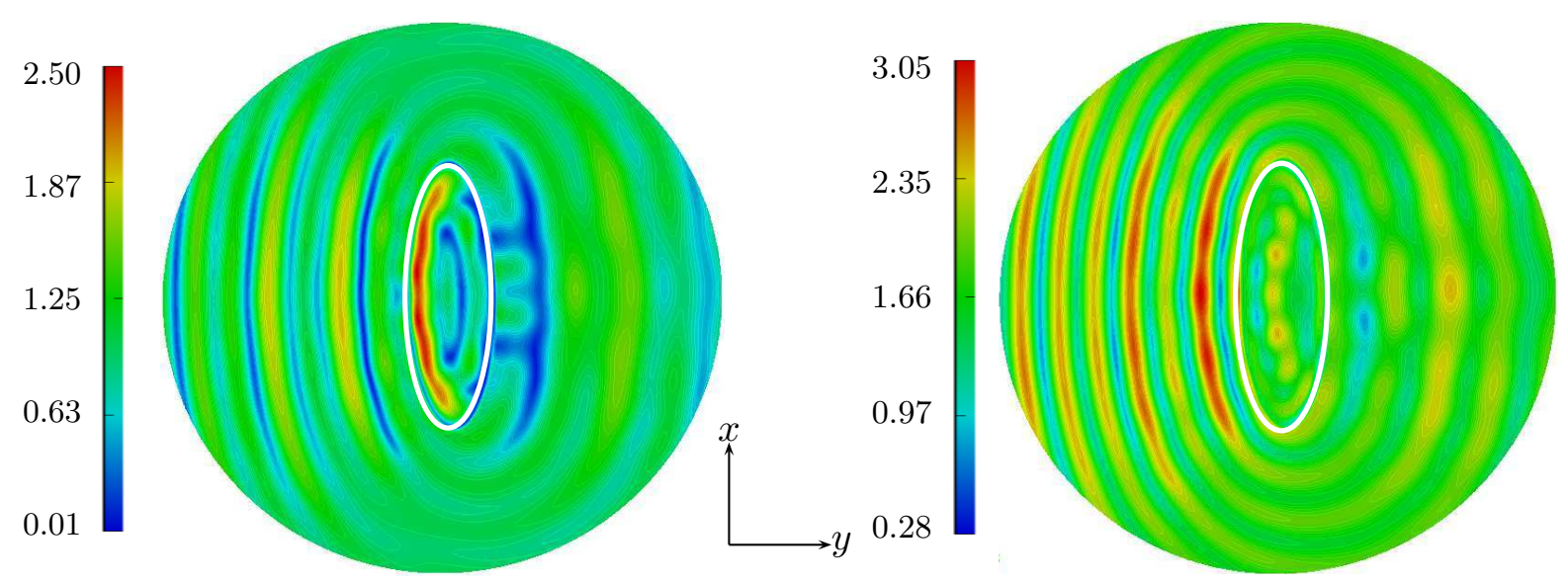

Figure 22: Diffraction of an oblique incident $P$ plane wave by a semi-ellipsoidal canyon: horizontal (left) and vertical (right) computed displacement on canyon surface and meshed part of free surface (normalized frequency $k_{S} a / \pi=2$ ). The white ellipse depicts the canyon edge.

points A, B, C, D, E defined on Fig. 18), are in good agreement. The present computation (featuring a truncation radius $D=6 a$ and a leaf level $\bar{\ell}=3$ ) required 11 GMRES iterations and 9s of CPU time per iteration. Finally, results obtained using the present FM-BEM for a higher frequency defined by $k_{\mathrm{S}} a / \pi=2$ are presented in terms of the $y$ and $z$ components of the displacement field (Fig. 22). The problem size is $N=353232$. The computation, performed with a leaf level $\bar{\ell}=5$, required 32 GMRES iterations and 143s of CPU time per iteration.

\section{Conclusions}

In this article, the Fast Multipole Method has been succesfully extended to $3 D$ elastodynamics in the frequency domain. Combined with the BEM formulation, it permits to reduce the computational burden, in both CPU time and memory requirements, for the analysis of wave propagation (e. g. seismic), and allows to run BEM models of size $N=O\left(10^{6}\right)$ on an ordinary PC. Comparisons with analytical or previously published numerical results show the efficiency and accuracy of the present elastodynamic FM-BEM. Theoretical complexity estimates for both the single-level and multi-level formulations were derived and corroborated by numerical experiments.

Applications of the present FM-BEM to realistic cases in seismology are under way. Moreover, a natural extension of this work consists in formulating multipole expansions of other fundamental solutions, with the half-space elastodynamic fundamental solution being currently investigated. Finally, improving the efficiency of the elastodynamic FM-BEM also requires further research into refined (direct/inverse) extrapolation techniques (for lowering the $O\left(N^{3 / 2}\right)$ of this step) and a well-chosen preconditioning strategy (for reducing the GMRES iteration count).

\section{References}

[1] Abramowitz, M., Stegun, I.A. Handbook of mathematical functions with formulas, graphs, and mathematical tables. Dover (1991).

[2] Alléon, G., Benzi, M., Giraud, L. Sparse approximation inverse preconditioning for dense linear systems arising in computational electromagnetics. Numer. Algor., 16:1-15 (1997).

[3] Bonnet, M. Boundary Integral Equation Method for Solids and Fluids. Wiley (1999). 
[4] Brebbia, C. A., Telles, J. C. F., Wrobel, L. C. Boundary element techniques. Springer (1984).

[5] Cheng, H., Crutchfield, W. Y., Gimbutas, Z., Greengard, L. F., Ethridge, J. F., Huang, J., Rokhlin, V., Yarvin, N. A wideband fast multipole method for the Helmholtz equation in three dimensions. J. Comp. Phys., 216:300-325 (2006).

[6] Cruse, T.A. Numerical solutions in three-dimensional elastostatics. Int. J. Solids Struct., 5:12591274 (1969).

[7] Dangla, P., Semblat, J. F., Xiao, H., Delépine, N. A simple and efficient regularization method for 3D BEM: application to frequency-domain elastodynamics. Bull. Seism. Soc. Am., 95:19161927 (2005).

[8] Darve, E. The Fast Multipole Method : Numerical Implementation. J. Comp. Phys., 160:195240 (2000).

[9] Darve, E. The fast multipole method: I. Error analysis and asymptotic complexity. SIAM J. Numer. Anal., 38:98-128 (2000).

[10] Darve, E., Havé, P. A fast multipole method for Maxwell equations stable at all frequencies. Phil. Trans. Roy. Soc. (London), A362:603-628 (2004).

[11] Epton, M.A., Dembart, B. Multipole translation theory for the three-dimensional Laplace and Helmholtz equations. SIAM J. Sci. Comp., 16:865-897 (1995).

[12] Eringen, A.C., Suhubi, E.S. Elastodynamics, vol. II-linear theory. Academic Press (1975).

[13] Eshraghi, H., Dravinski, M. Scattering of plane harmonic SH, SV, P and Rayleigh waves by nonaxisymmetric three-dimensional canyons: a wave function expansion approach. Earthquake Engng. and Struct. Dyn., 18:983-998 (1989).

[14] Fischer, M., Gaul, L. Application of the fast multipole BEM for structural-acoustic simulations. J. Comput. Acoust., 13:87-98 (2005).

[15] Frayssé, V., Giraud, L., Gratton, S., Langou, J. A Set of GMRES Routines for Real and Complex Arithmetics. http://www. cerfacs.fr/algor/Softs/GMRES/ (2007).

[16] Fujiwara, H. The fast multipole method for the integral equations of seismic scattering problems. Geophys. J. Int., 133:773-782 (1998).

[17] Fujiwara, H. The fast multipole method for solving integral equations of three-dimensional topography and basin problems. Geophys. J. Int., 140:198-210 (2000).

[18] Givoli, D. Numerical Methods for Problems in infinite domains. Elsevier (1992).

[19] Greengard, L. The rapid evaluation of potentiel fields in particle systems. MIT Press (1988).

[20] Greengard, L., Rokhlin, V. A fast algorithm for particle simulations. J. Comp. Phys, 73:325-348 (1987).

[21] Gumerov, N. A., Duraiswami, R. Fast multipole methods for the Helmholtz equation in three dimensions. Elsevier (2005).

[22] Guzina, B. B., Pak, R. Y. S. On the Analysis of Wave Motions in a Multi-Layered Solid. Quart. J. Mech. Appl. Math., 54:13-37 (2001). 
[23] Hackbusch, W., Nowak, Z. P. On the fast matrix multiplication in the boundary element method by panel clustering. Numer. Math., 54:463-491 (1989).

[24] Ihlenburg, F., Babuška, I. Dispersion analysis and error estimation of Galerkin finite element methods for the Helmholtz equation. Int. J. Numer. Meth. Engng., 38:3745-3774 (1995).

[25] Jiang, L. J., Chew, W. C. A mixed-form fast multipole algorithm. IEEE Trans. Antennas Propagat., 53:4145-4156 (2005).

[26] Komatitsch, D., Vilotte, J. P. The spectral element method: an efficient tool to simulate the seismic response of 2D and 3D geological structures. Bull. Seism. Soc. Am., 88:368-392 (1998).

[27] Kurz, S., Rain, O., Rjasanow, S. The adaptive cross approximation technique for the 3D boundary element method. IEEE Transactions on Magnetics, 38:421-424 (2002).

[28] Lee, V. W. 3D diffraction of plane P-, SV- and SH-waves by a hemispherical alluvial valley. Soil. Dyn. Earthquake Engng., 3:133-144 (1984).

[29] Liao, W.I., Teng, T.J., Yeh, C.S. A series solution and numerical technique for wave diffraction by a three-dimensional canyon. Wave Motion, 39:129-142 (2004).

[30] Liu, Y., Nishimura, N., Otani, Y., Takahashi, T., Chen, X. L., Munakata, H. A fast boundary element method for the analysis of fiber-reinforced composites based on a rigid-inclusion model. ASME J. Appl. Mech., 72:115-128 (2005).

[31] Lu, C.C, Chew, W.C. Fast algorithm for solving hybrid integral equations. Microwaves, Antennas and Propagation, IEE Proc. H, 140:455-460 (1993).

[32] Lu, C.C, Chew, W.C. A multilevel algorithm for solving a boundary integral equation of wave scattering. Microwave Opt. Technol. Lett., 7:466-470 (1994).

[33] Mantic, V. A new formula for the C-matrix in the Somigliana identity. J. Elast., 33:191-201 (1993).

[34] Moczo, P., Kristek, J., Vavrycuk, V., Archuleta, R.J., Halada, L. 3D heterogeneous straggeredgrid finite-difference modelling of seismic motion with volume harmonic and arithmetic averaging of elastic moduli and densities. Bull. Seism. Soc. Am., 92:3042-3066 (2002).

[35] Nédélec, J. C. Acoustic and electromagnetic equations. Applied mathematical sciences (vol. 144). Springer Verlag (2001).

[36] Nemitz, N., Bonnet, M. Topological sensitivity and FMM-accelerated BEM applied to 3D acoustic inverse scattering. Engng. Anal. Bound. Elem. (2007, accepted).

[37] Nishimura, N. Fast multipole accelerated boundary integral equation methods. Appl. Mech. Rev., 55(4) (2002).

[38] Ohnuki, W. C., Chew, W. C. Error minimization of multipole expansion. SIAM J. Sci. Comput., 26:2047-2065 (2005).

[39] Rao, S., Wilton, D., Glisson, A. Electromagnetic scattering by surfaces of arbitrary shape. IEEE Trans. Antennas Propag., 30:409-418 (1982).

[40] Reinoso, E., Wrobel, L. C., Power, H. Three-dimensional scattering of seismic waves from topographical structures. Soil. Dyn. Earthquake Engng., 16:41-61 (1997). 
[41] Rizzo, F.J. An integral equation approach to boundary value problems of classical elastostatics. Quart. Appl. Math., 25:83-95 (1967).

[42] Rokhlin, V. Rapid solution of integral equations of classical potential theory. J. Comp. Phys., 60:187-207 (1985).

[43] Rokhlin, V. Rapid solution of integral equations of scattering theory. J. Comp. Phys., 86:414439 (1990).

[44] Rokhlin, V. Diagonal forms of translation operators for the Helmholtz equation in three dimensions. Appl. Comp. Harmonic Anal., 1:82-93 (1993).

[45] Rokhlin, V. Sparse diagonal forms for translation operators for the Helmholtz equation in two dimensions. Appl. Comp. Harmonic Anal., 5:36-67 (1998).

[46] Saad, Y. Iterative methods for sparse linear systems. SIAM (2003).

[47] Saad, Y., Schultz, M.H. GMRES: a generalized minimal residual algorithm for solving nonsymmetric linear systems. SIAM J. Sci. Stat. Comput., 7:856-869 (1986).

[48] Sakuma, T., Yasuda, Y. Fast multipole boundary element method for large-scale steady-state sound field analysis. Part I: setup and validation. Acta Acustica united with Acustica, 88:513525 (2002).

[49] Sánchez-Sesma, F. J. Diffraction of elastic waves by 3D surface irregularities. Bull. Seism. Soc. Am., 73:1621-1636 (1983).

[50] Sebert, A. F., Soenarko, B., Rizzo, F. J., Shippy, D. J. An advanced computational method for the radiation and scattering of acoustic waves in three dimensions. J. Acoust. Soc. Am., 77:362-368 (1985).

[51] Semblat, J. F., Brioist, J.J. Efficiency of higher order finite elements for the analysis of seismic wave propagation. J. Sound Vib., 231:460-467 (2000).

[52] Song, J., Lu, C.C., Chew, W.C. Multilevel fast multipole algorithm for electromagnetic scattering by large complex objects. IEEE Trans. Antennas Propag., 42:1488-1493 (1997).

[53] Sylvand, G. La méthode multipôle rapide en éléctromagnétisme : performances, parallélisation, applications. Ph.D. thesis, ENPC, Paris, France (2002).

[54] Takahashi, T., Nishimura, N., Kobayashi, S. A fast BIEM for three-dimensional elastodynamics in time domain. Engng. Anal. Bound. Elem., 27:491-506 (2003).

[55] Tausch, J. Sparse BEM for potential theory and Stokes flow using variable order wavelets. Comp. Mech., 32:312-3185 (2003).

[56] Ying, L., Biros, G., Zorin, D. A kernel-independent adaptive fast multipole in two and three dimensions. J. Comp. Phys., 196:591-626 (2004).

[57] Yokoi, T. The high order Born approximation applied to improve the solution of seismic response of a three-dimensional canyon by the Indirect Boundary Method. Physics of the Earth and Planetary Interior, 137:97-106 (2003).

[58] Yoshida, K.I. Applications of fast multipole method to boundary integral equation method. Ph.D. thesis, University of Kyoto (2001). 